OPEN ACCESS

Edited by:

Hao Zhang,

Jinan University, China

Reviewed by:

Andrew Skildum,

University of Minnesota, United States

Tang-Long Shen,

National Taiwan University, Taiwan

*Correspondence:

Yongmei Yin

ymyin@njmu.edu.cn

Wei Li

real.Iw@163.com

Specialty section:

This article was submitted to

Molecular and Cellular Oncology,

a section of the journal

Frontiers in Cell and Developmental

Biology

Received: 26 June 2021 Accepted: 27 September 2021 Published: 01 November 2021

Citation:

Wang $X$, Sun C, Huang $X, L i J$, Fu Z, Li W and Yin Y (2021) The Advancing Roles of Exosomes in Breast Cancer. Front. Cell Dev. Biol. 9:731062. doi: 10.3389/fcell.2021.731062

\section{The Advancing Roles of Exosomes in Breast Cancer}

\author{
Xi Wang ${ }^{1,2}$, Chunxiao Sun ${ }^{1}$, Xiang Huang ${ }^{1}$, Jun Li ${ }^{1}$, Ziyi Fu',3, Wei Li ${ }^{1 *}$ and Yongmei Yin ${ }^{1 *}$ \\ ${ }^{1}$ Department of Oncology, The First Affiliated Hospital of Nanjing Medical University, Nanjing, China, ${ }^{2}$ The First School \\ of Clinical Medicine, Nanjing Medical University, Nanjing, China, ${ }^{3}$ Nanjing Maternity and Child Medical Institute, Obstetrics \\ and Gynecology Hospital, Nanjing Medical University, Nanjing, China
}

Breast cancer $(\mathrm{BC})$ develops from breast tissue and is the most common aggressive malignant tumor in women worldwide. Although advanced treatment strategies have been applied and reduced current mortality rates, BC control remains unsatisfactory. It is essential to elucidate the underlying molecular mechanisms to assist clinical options. Exosomes are a type of extracellular vesicles and mediate cellular communications by delivering various biomolecules (oncogenes, oncomiRs, proteins, and even pharmacological compounds). These bioactive molecules can be transferred to change the transcriptome of target cells and influence tumor-related signaling pathways. Extensive studies have implicated exosomes in BC biology, including therapeutic resistance and the surrounding microenvironment. This review focuses on discussing the functions of exosomes in tumor treatment resistance, invasion and metastasis of BC. Moreover, we will also summarize multiple interactions between exosomes and the $\mathrm{BC}$ tumor microenvironment. Finally, we propose promising clinical applications of exosomes in $\mathrm{BC}$.

Keywords: exosomes, breast cancer, tumor microenvironment, resistance, clinical applications

\section{INTRODUCTION}

\section{Breast Cancer}

According to Globocan estimates, breast cancer (BC) is one of the most commonly diagnosed cancers in both sexes combined and is the leading cause of cancer death among females. New data published in 2020 suggest that $30 \%$ of women in United States will develop BC during their lifetime, and 15\% of patients will die from it (Siegel et al., 2020). In addition, it has a morbidity rate of $27.8 \%$ and a $15 \%$ mortality rate in women worldwide according to a global cancer estimate (Bray et al., 2018).

Breast cancer is a heterogeneous disease that exhibits extensive genomic, transcriptomic, and proteomic dysregulation. With diverse gene expression of steroid hormone receptors [estrogen receptors (ER), progesterone receptor (PR)] and human epidermal growth factor receptor 2 (HER2), and BC is classified into four molecular subtypes. Such heterogeneity has resulted in marked differences in therapeutic options (Harbeck and Gnant, 2017; Table 1).

\section{Exosomes}

Exosomes are a separate type of endosome-released nanometer-sized extracellular vesicles released by all kinds of eukaryotic cells. Vesicles originating from various cells are classified into two main types, exosomes and ectosomes, all of which are cell-derived vesicles that are enclosed 
TABLE 1 | Different classifications of BC.

\begin{tabular}{|c|c|c|c|}
\hline Subtypes & Classification & HER2 & Hormone receptor \\
\hline \multirow[t]{2}{*}{ HR positive and HER2 negative } & Luminal A-like & HER2 negative & ER or PR positive, or both, low proliferation (low Ki67) \\
\hline & Luminal B-like & HER2 negative & ER or PR positive, or both, high proliferation (high Ki67) \\
\hline \multirow[t]{2}{*}{ HER2-enriched } & Non-luminal & HER2 positive & ER and PR negative \\
\hline & Luminal & HER2 positive & ER or PR positive, or both \\
\hline Triple-negative & TNBC & HER2 negative & ER and PR negative \\
\hline
\end{tabular}

by lipid bilayers, with diameters ranging from 30 to $2,000 \mathrm{~nm}$ (Kalluri and LeBleu, 2020). The process of exosome generation and secretion is very complicated. Here, we briefly introduce and compare differences in this sophisticated procedure between exosomes and ectosomes. Exosomes are biopolymers that range from 30 to $100 \mathrm{~nm}$ in diameter, have unique structural and mechanical characteristics and are formed by two invaginations of the plasma membrane. The first invagination occurs along biomolecules associated with the extracellular milieu and forms an intracellular cup-shaped structure called the early-sorting endosomes, which subsequently mature into late-sorting endosomes. Intracellular multivesicular bodies containing intraluminal vesicles are formed in the subsequent invagination after receiving late-sorting endosomes. From early to terminal endosome maturation, multivesicular bodies are processed either by fusing with the plasma membrane to undergo exocytosis of the contained intraluminal vesicles (namely, exosomes) into the extracellular space or fusing with lysosomes or autophagosomes to be degraded (Kalluri and LeBleu, 2020). In contrast to exosomes, ectosomes, including microvesicles, microparticles, and large vesicles, bud directly and pinch off from the plasma membrane and are extremely heterogeneous in size (Cocucci and Meldolesi, 2015).

Exosomes are distributed through human body fluids to transport their cargos to cells in the vicinity or dwelling remotely, which brings notable advantages to detecting potential pathological conditions. The uptake mechanisms of exosomes are widely discussed, including direct plasma membrane fusion and endocytosis, such as micropinocytosis (Nakase et al., 2015) and phagocytosis (Feng et al., 2010).

Exosomes are secreted by almost every kind of cell and play an important role in cell-cell communication (van Niel et al., 2018). They contain and deliver numerous biomolecules, ranging from multiple proteins, lipids, DNA, microRNAs (miRNAs), circular RNAs (circRNAs), messenger RNAs (mRNAs), long non-coding RNAs (lncRNAs), and metabolites, to target cells. Intriguingly, exosome contents may vary, as distinct proteins and nucleic acids are enclosed according to their cell of origin (Wen et al., 2019). However, the selection mechanisms for content loading remain unveiled. In terms of cancers, an increasing number of articles have reported exosome involvement in cancer development and function and as potential vectors to aid in improving therapeutic strategies. Exosomes may also have profound impacts on the tumor microenvironment, as they represent intensively specialized entities of interaction carrying specific surface markers, oncogenic proteins and other biomolecules that can be transferred horizontally into stromal cells. Consequently, the tumor microenvironment is altered to allow for tumor progression (Rak and Guha, 2012). As it is difficult to assign the correct nomenclature to lipid bilayer particles secreted by cells in diverse experimental conditions, according to the claim of the "International Society for Extracellular Vesicles" (Théry et al., 2018), some scientific reports referred to "extracellular vesicles" as an unified term covering small extracellular vesicles containing exosomes. Thus, in this present review, several findings related to extracellular vesicles in the discussion of metastatic BC were also presented.

\section{Tumor Microenvironment}

The tumor microenvironment often refers to a dynamic region intrinsic to the solid tumor. In addition to tumor cells, it also embodies multiple cell types and non-cancerous components such as fibroblasts, immune-related cells (e.g., tumor associated macrophages, dendritic cells, mesenchymal stem cells, and $\mathrm{T}$ cells), adipocytes and endothelial cells. These cells each with diverse biological contributions constitute an integral part of the unique microenvironment for BC cells. Meanwhile, they have crucial roles in local interact with $\mathrm{BC}$ progression. For example, cancer-associated fibroblasts are believed to suppress $\mathrm{BC}$ microenvironment immunity and was exemplified in the work undertaken by Costa et al. (2018). One of the four cancerassociated fibroblasts subsets which called CAF-S1, inhibited T effector proliferation by enhancing the regulatory $\mathrm{T}$ cell capacity.

Also, the tumor microenvironment provides a milieu for communication between cancer cells and surrounding immune cells, fibroblasts, endothelial cells, and adipocytes. Interactions between malignant cells and non-malignant cells in the tumor microenvironment occur via exosomes, soluble cytokines or signaling molecules that cancer cells are capable of manipulating to their own advantage in processes such as angiogenesis, immune evasion, migration, metastasis, and drug resistance (Meads et al., 2009). Under the modulation of the tumor microenvironment, non-tumor cells can be affected by tumorrelated active substances, forcing them to constantly adapt, and creating an environment that facilitates tumor growth (Quail and Joyce, 2013). For example, normal fibroblasts can be stimulated to become cancer-associated fibroblasts and create a specific extracellular matrix to support tumor cell migration (Chen et al., 2017a). Diverse immune and inflammatory molecules in the tumor microenvironment also block the immune killing response, empowering tumor cell survival (Toor et al., 2019). In addition, the hypoxic tumor microenvironment that is generated and its primary effectors represent a critical step in the tumorigenic process (Petrova et al., 2018). Together, these 
cancer-associated activities in the tumor microenvironment play detrimental roles in favoring neoplasm malignant development (Jarosz-Biej et al., 2019).

In this review, we summarize the multifaceted functions of exosomes in BC therapeutic resistance, invasion, and mediation of the tumor microenvironment to better understand and treat related malignant progression. In addition, their potential applications in diagnosing and providing novel clinical therapeutic options are also discussed in the section "Conclusion."

\section{THE EFFECTS OF EXOSOMES IN THE BREAST CANCER TUMOR MICROENVIRONMENT}

The tumor microenvironment is often described as the soil where tumor cells grow, which is formed by a variety of normal cells, signal-transmitting molecules, blood vessels, and extracellular matrix (Quail and Joyce, 2013). Consequently, cancer and other cells dwelling in the same niche are involved in multiple modes of interactions to synchronize and antagonize the negative influence of environmental changes, such as drug resistance (Diaz Bessone et al., 2019). In detail, surrounding normal cells, including immune-associating cells, vascular endothelial cells, adipocytes, fibroblasts, and bone-marrow mesenchymal stromal cells, are recruited to and have prominent roles in cancer (Kalluri and Zeisberg, 2006). For instance, epithelial-mesenchymal transition is necessary during embryonic development. However, findings found that various alterations in glycolysis, oxidative phosphorylation, and autophagy in this process promoted tumor multidrug resistance (Erin et al., 2020). Many tumor cells survive by developing immune evasion mechanisms, including secretion of neoplastic proteins, escape from T-cell attack, promotion of $\mathrm{T}$ regulatory cells, and decreased levels of antigen-presenting proteins. Studies have revealed that BC cell-released exosomes are capable of affecting the immune system through interplay with T-cells, regulatory T-cells, dendritic cells, and macrophages.

\section{The Effects of Exosomes on Immune System Regulation}

Immunoediting in cancer is a dynamic process, which further determines tumor progression. The distinct modes between immune cells and cancer cells result in widely different outcomes. Immune cells like dendritic cells play a central role in immune responses against cancer. On the contrary, infiltration by plasmacytoid dendritic cells was found correlates with decreased overall survival and poor relapse-free survival in BC (Treilleux et al., 2004). Besides, macrophages- 2 released CHI3L1 was found prompted tumor progression, which revealed a novel field of macrophages with respect to BC metastasis (Chen et al., 2017b). In this part, we will focus on describing the effects of exosomes on BC immune system regulation. Tumor-derived exosomes contain molecules that can stimulate immune cell dysfunction and transform the surrounding microenvironment into a suitable niche conducive to their growth and metastasis (Figure 1).
Recently, one study revealed that lncRNAs in exosomes secreted from BC were correlated with macrophage polarization. Specifically, lncRNA BCRT1 mediated by exosomes promoted M2 polarization of macrophages, which further accelerated BC progression (Liang et al., 2020). Similarly, a previous work also demonstrated that macrophage polarization in the cancer-related microenvironment was altered by BC-derived exosomes, partially through the glycoprotein 130-STAT3 signaling pathway (Ham et al., 2018). Furthermore, the progression of early-stage myeloidderived suppressor cells was promoted by tumor exosomederived miR-9 and miR-181a by activating the JAK/STAT signaling pathway in BC (Jiang et al., 2020).

Meanwhile, the function of exosomes that arise from the tumor microenvironment on the malignant progression of $\mathrm{BC}$ cells is also significant (Figure 2). It was demonstrated that cancer-related macrophages in the tumor microenvironment contributed to enhanced BC cell aerobic glycolysis and apoptotic resistance by transmitting extracellular vesicles encompassing a distinctive lncRNA. This HIF- $1 \alpha$-stabilizing lncRNA was clinically positively associated with poor chemotherapeutic responses and shorter survival in BC patients (Chen et al., 2019). In addition, $\mathrm{BC}$ cells can reside in the bone marrow and remain dormant for decades. However, how macrophages modulate dormancy is still poorly understood. One study conducted a survey and found that exosomes released by macrophages exhibiting an M1 phenotype activated NF- $\kappa \mathrm{B}$ to reverse silent $\mathrm{BC}$ cells into cycling cells, which contributed to BC progression (Walker et al., 2019). Another study concluded that macrophages played an important role in the regulation of BC metastasis by transmitting exosome-mediated invasion-potentiating miRNA (Yang et al., 2011).

Immunological surveillance plays a vital role in the generation and maintenance of the anti-tumor function of the immune system (Schreiber et al., 2011), and studies have sought to investigate the role of exosomes in its development and subsequent outcomes. For instance, Yang et al. (2018) demonstrated that BC-released exosomes were capable of blunting T-cell activation and ablating activities by expressing programmed cell death-1 on their surface, effectively helping tumor cells evade immune surveillance. After bypassing the immune system, tumor cells are potentiated to successfully seed and colonize various distant organs, such as the lung. Exosomes secreted by highly metastatic murine BC cells inhibit anti-tumor immune responses in premetastatic organs, directly suppressing T-cell proliferation and suppressing NK cell cytotoxicity. Hence, this process contributes to promoting metastasis (Wen et al., 2016). A recent finding reported that a unique IncRNA, SNHG16, enclosed by exosomes was secreted from BC cells and induced CD73 $+\gamma \delta \mathrm{T} 1$ cells to act as immunosuppressive regulatory T-cells by activating the TGF- $\beta 1 /$ SMAD5 pathway (Ni et al., 2020).

As discussed above, the $\mathrm{BC}$ tumor microenvironment contains specific immune sub-populations such as immune surveillance evasion and immune regulation contributed to inhibited antitumor immune response of host cells. Several anti-inflammatory mechanisms presented by reciprocal interactions between BC cells and tumor microenvironment by utilizing exosomes as 


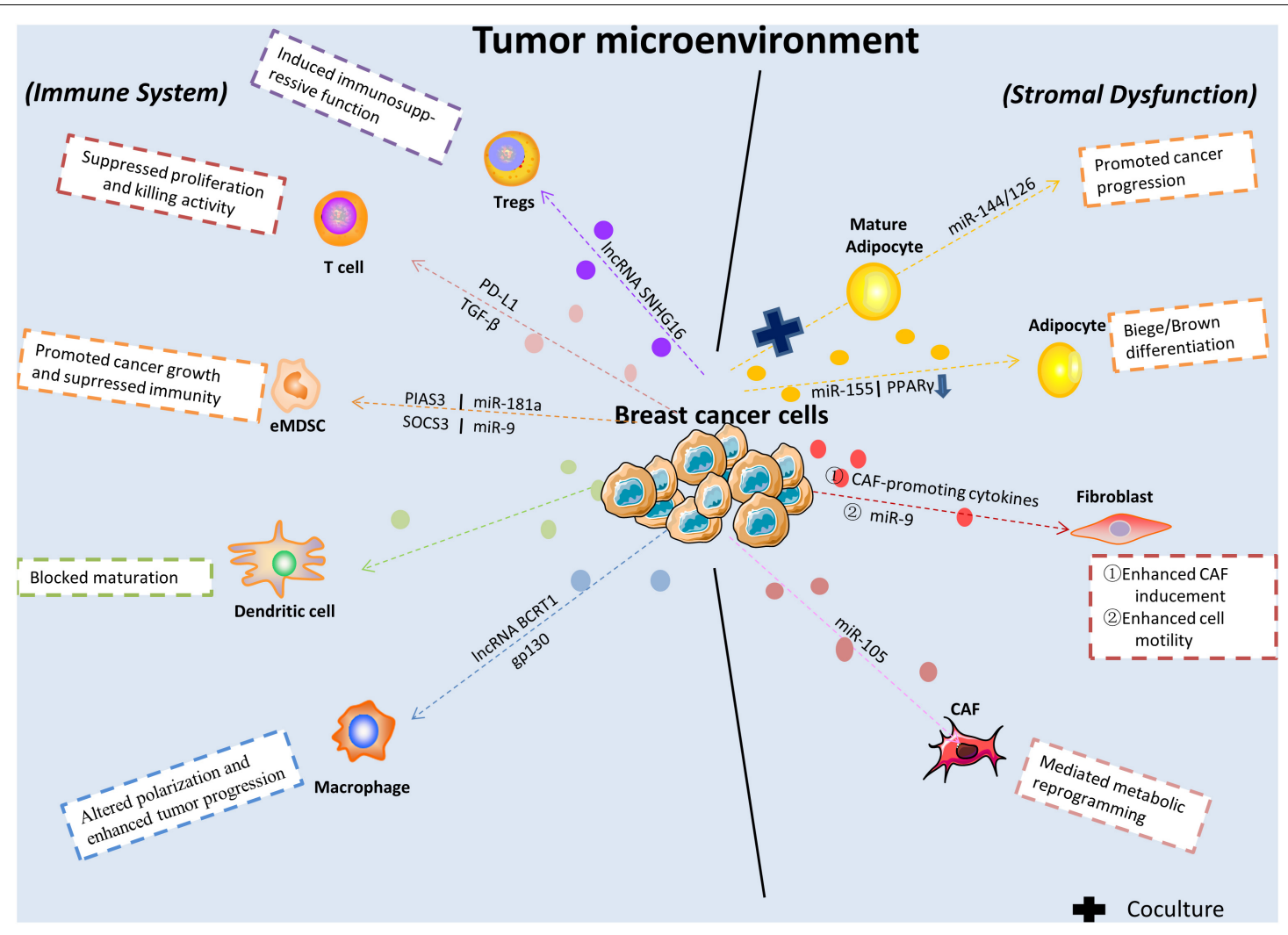

FIGURE 1 | Breast cancer-released exosomes in regulating immune system, fibroblasts, and adipocytes in tumor microenvironment. BC-derived exosomes are released to alter immune system and cause stromal cell dysfunction in tumor microenvironment via transferring multifarious cargos and targeting genes or signaling pathways. eMDSC, early-stage myeloid-derived suppressor cells; CAF, cancer associated-fibroblast; Tregs, regulatory $T$ cells.

mediators have been summarized. In section "Conclusion," the above findings demonstrated that blocking cancer-derived exosomes may have favorable impacts for treating BC.

\section{The Effects of Exosomes on Fibroblast Cells}

Fibroblasts are known as non-vascular, non-inflammatory, and non-epithelial cells in surrounding tissue. Their secretion of growth factors, various chemokines and extracellular matrix facilitates recruiting the endothelial cells and pericytes. They are also found activated into cancer-associated fibroblasts and become a key determinant in the malignant progression of tumor including metastasis, invasion, resistance, and imply an important target in cancer treatments (Plava et al., 2019). As pioneering studies suggested that tumor cells can be promoted in an wound-repair-associated microenvironment. It is vital for us to deepen our understanding in the field of reciprocal effects between fibroblast and tumor microenvironment (Bissell and Hines, 2011). A recent finding revealed the PERK signaling was selectively activated by Rho-associated kinase which caused fibroblast reprogramming and contributed to BC progression (Boyle et al., 2020). Furthermore, fibroblast-expressed PIK3C $\delta$ was found promoting TNBC progression with the help of a high-throughput siRNA kinome screening technology and recognized as a potential target (Gagliano et al., 2020). The tumor microenvironment forms the core site in which neoplastic cells, stromal fibroblasts and cancer-associated fibroblasts interact with each other, and exosomes play a complex role in that interplay (Figure 2). For example, endogenous RNAs must remain shielded under non-pathological conditions. However, one study showed that RN7SL1, an endogenous RNA normally covered by RNA binding proteins SRP9/14, was increased in stromal fibroblast exosomes in response to triggering stromal NOTCH-MYC. After being transferred to ISG-R BC cells in exosomes and then bound to RIG-I, this unshielded RN7SL1 exosomal RNA promoted aggressive features of cancer (Nabet et al., 2017). Additionally, a study demonstrated that stromal fibroblasts are converted into cancer-associated fibroblasts with low expression of p85 $\alpha$. Furthermore, p85 $\alpha$-deficient fibroblastderived exosomes carrying the Wnt10b protein were transferred into BC cells, which induced epithelial-to-mesenchymal transition and was consequently involved in breast malignant progression (Chen et al., 2017a). Another study established that the horizontal transfer of exosomal mitochondrial DNA derived from cancer-associated fibroblasts exerted its function as a tumorigenesis signal in hormonal therapy-resistant disease. The specific mechanisms underlying the transfer include promoting escape from metabolic quiescence of therapy-mediated cancer stem-like cells and further contributing to hormonal therapy 


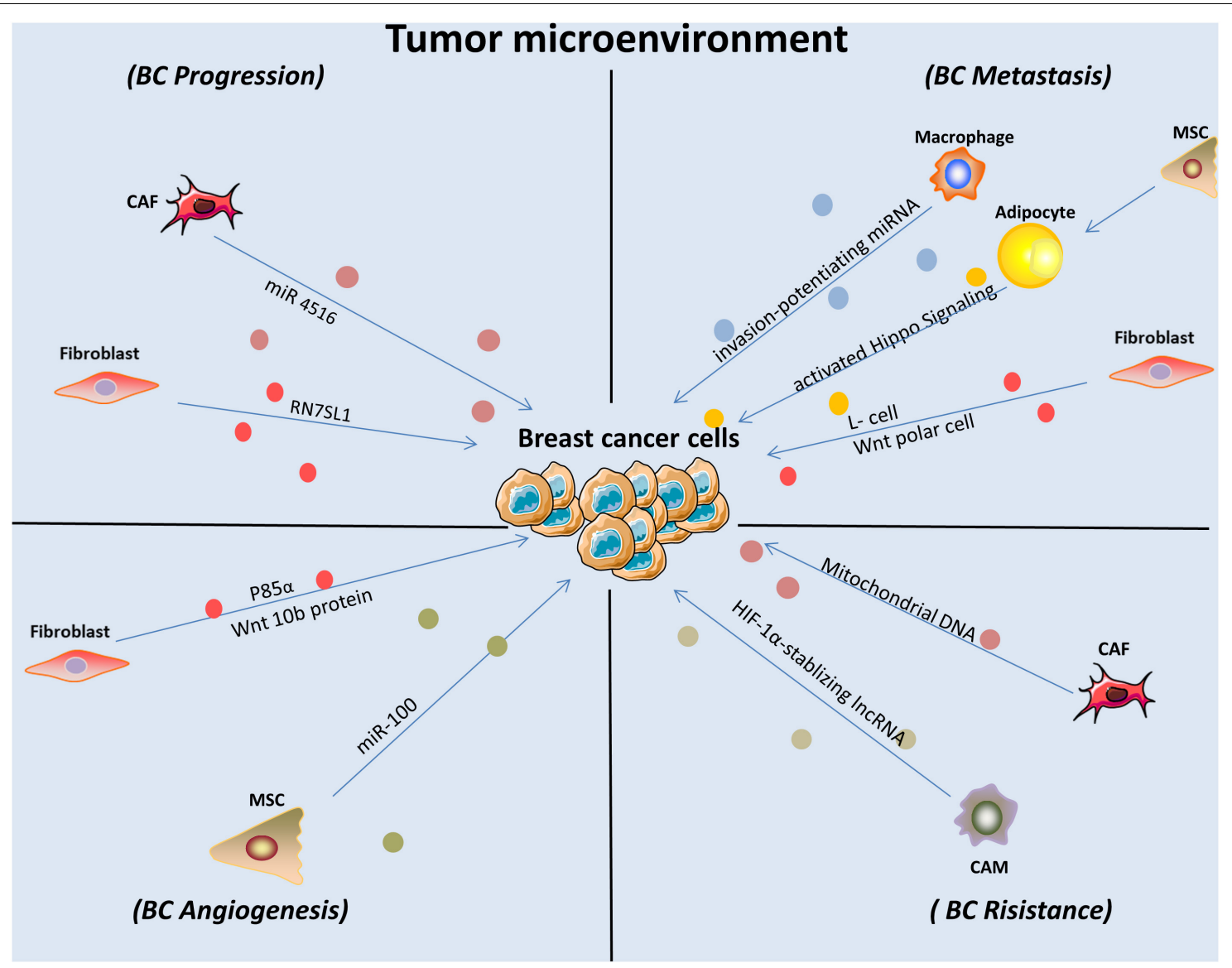

FIGURE 2 | Exosomes derived from stromal cells affected BC cell behaviors and contributed to enhanced malignant progression. CAM, cancer associated macrophage; MSC, mesenchymal stem cells; CAF, cancer associated fibroblast.

resistance in oxidative phosphorylation-dependent BC cells (Sansone et al., 2017b). In addition, a specific exosomal miRNA4516 was recently found to be involved in intercellular crosstalk. The miRNA secreted from cancer-associated fibroblasts was correlated with the regulation of triple-negative BC (TNBC) progression. Specifically, reduced expression of miRNA-4516 promoted proliferation and malignancy in TNBC by targeting FOSL1, which exerted its function as a tumor progression mediator and thus contributed to tumor development (Kim et al., 2020). A recent study that centered on the relationship between cancer-associated fibroblasts and BC cell activities was conducted in a $\mathrm{BC}$ mouse model and demonstrated that after ablation of focal adhesion kinase, expression of exosomal miRNA-16, and 148a was increased in cancer-associated fibroblasts, which inhibited migration and metastasis in $\mathrm{BC}$ (Wu et al., 2020). A previous study established that exosomes derived from a fibroblast cell line, L cells, stimulated BC cell lines (MDA-MB-231 and SUM-159PT) to exhibit protrusive activity, motility, and lung metastasis through the Wnt-planar cell polarity pathway. Specifically, it was revealed by the authors in subsequent studies that fibroblast-released exosomes colocalized with BC cell-derived Wnt11 within endocytic vesicles after uptake in target cells, which was vital for enhancing exosome-stimulated motility in BC cell lines (Luga et al., 2012). This direct evidence demonstrated that exosomes play a part in mediating metastatic properties between stromal and BC cells.

Another investigation found that exosomal miRNA-9 was transmitted from $\mathrm{BC}$ cells to normal fibroblasts and resulted in enhanced cell motility. Furthermore, this miRNA was also released by fibroblasts and was in turn capable of altering tumor cell behavior via extracellular matrix remodeling pathways (Baroni et al., 2016). Additionally, extracellular vesicles derived from BC containing miRNA-105 activated oncoprotein MYC signal transduction. This result triggered metabolic reprogramming of cancer-associated fibroblasts by conditioning their metabolic features in different metabolic environments and sustained tumor growth (Yan et al., 2018; Figure 1).

The underlying mechanism of how normal fibroblasts were reached and transformed by cancer-associated fibroblastsderived promoting cytokines is unclear. Closer investigation into this question found that promoting cytokines gained the ability to effectively break the extracellular matrix barrier after loading into exosomes and reached normal fibroblasts during the early stages of BC development. Diffusion of exosomes was enhanced with the aid of extracellular matrix fibril alignment in a forcedependent manner (Jung et al., 2020). 


\section{The Effects of Exosomes on Angiogenesis}

Mesenchymal stem cells was first described as multipotent hematopoietic bone marrow cells to give rise to bone, marrow stroma, adipocytes, muscle, and connective tissue (Caplan, 1991). Reduction of tumor growth by mesenchymal stem cells can be modulated by inhibiting angiogenesis, P2X signaling was implied as a pathway promoting mesenchymal stem cells mediated BC cell proliferation (Maffey et al., 2017). Endothelial cells are also important players in the angiogenic promotion of cancer growth and metastatic spreading. A study found that the exchange of growth factors between co-cultured endothelial and BC cells are capable of modulating the angiogenic response and tumor invasion ability directly (Zhang et al., 2018). The shuttling of miRNA-100 from human mesenchymal stem cells to BC cells via exosomes, which suppressed angiogenesis in recipient cells, was substantiated by a previous study. This process was induced by modulating the mTOR/HIF-1 $\alpha$ /VEGF signaling axis in BC cells (Pakravan et al., 2017). Annexin II was identified as one of the most highly encapsulated proteins in exosomes using proteomics profiling and was found to be significant in BC pathogenesis. One study demonstrated that exosomal Annexin II promoted tPAdependent angiogenesis both in vitro and in vivo, probably by functioning as a specific receptor for both tPA and plasminogen (Maji et al., 2017). Furthermore, the level of exosomal Annexin A2 was found elevated in the serum of African-American TNBC BC women, which also correlated with intensive possibility of promoting angiogenesis (Chaudhary et al., 2020). Mesenchymal stem cell-derived exosomes contain protein and RNA profiles that are different from their donor cells and can be internalized by $\mathrm{BC}$ cells. Upon delineating the mechanism, mesenchymal stem cell-derived exosomes contained a specific miRNA-16, which targeted vascular endothelial growth factor and drastically downregulated its expression. As a result, the inhibition of angiogenesis in $\mathrm{BC}$ was induced both in vitro and in vivo (Lee et al., 2013). After treatment with docosahexaenoic acid, expression of exosomal miRNAs was increased in MCF7 BC cells. Mechanistically, with the application of drug-treated BC-derived exosomal miRNAs to endothelial cell cultures, researchers found that tube formation was significantly decreased, suggesting that BC exosome signaling was targeted by docosahexaenoic acid to inhibit angiogenesis (Hannafon et al., 2015). In addition, a study focusing on the role of exosomes isolated from TNBC cells in cancer progression compared differences between exosomes secreted from a less invasive cell line. It was found that exosomes derived from more invasive TNBC cells promoted endothelial tubule formation after uptake, which positively correlated with vasculogenesis and subsequent angiogenesis in secondary cells (O’Brien et al., 2013).

\section{The Effects of Exosomes in the Interactions Between Adipocytes and Breast Cancer Cells}

It was demonstrated that the co-cultivation of $\mathrm{BC}$ cells with adipocytes contributed to elevated invading capacity both in vitro and in vivo (Wu et al., 2019a). According to recently reported review, abundant factors are participated in the pro-tumorigenic functions of adipocytes, including production of adipokines and other soluble factors (Choi et al., 2018). Also, obesity and adipocytes was reported strongly connected with poor prognosis of patients with BC (Picon-Ruiz et al., 2017). In this section we will give a brief summarize of the pivotal roles of adipocytes in BC cell growth and progression (Figure 2). A previous study demonstrated that exosomes derived from pre-adipocytes played important roles in regulating earlystage BC cell activities. After treatment with the natural anti-tumor compound shikonin, miRNA140 expression in exosomes increased and targeted SOX9 signaling, which may consequently block differentiation, stemness, and migration in the BC microenvironment (Gernapudi et al., 2015). It was also noted that adipocytes derived from mesenchymal stromal/stem cell-released exosomes promoted proliferation and migration after incorporation into the BC cell line MCF7 through the Hippo signaling pathway. Furthermore, these exosomes protected MCF7 cells from serum derivation or chemotherapyinduced apoptosis in vitro (Wang S. et al., 2019). A novel study investigated exosomes derived from cocultivation of BC cells with mature adipocytes and their effects on tumor progression. Mechanistically, researchers demonstrated that exosomal miRNA-144 and miRNA-126 were found induced beige/brown differentiation and reprogrammed metabolism in recipient adipocytes surrounding the tumor cells to facilitate BC progression (Wu et al., 2019b). In addition, cancer-related cachexia is a metabolic complication of cancer, and miRNA in exosomes secreted from BC cells plays a fundamental role in this process and consequently promotes cancer progression. $\mathrm{BC}$ cell-derived exosomes transferred miRNA-155 into adipocytes, where it exerted its function by promoting beige/brown differentiation and remodeling metabolism in resident adipocytes by downregulating PPAR $\gamma$ expression (Wu et al., 2018; Figure 1).

\section{Other Extracellular Vesicles and Cytokines in Regulating Tumor Microenvironment}

As exosomes are originated from extracellular vesicles which also play crucial roles in intercellular communications, in this paragraph we will focus on describing other extracellular vesicles and cytokines such as microvesicles, apoptotic bodies and their potential roles in regulating malignant process especially in $\mathrm{BC}$ tumor microenvironment. An investigation illuminated stromal microvesicles mediated evolution of cancer stemlike cells by transferring genetic material from local stromal cells to cancer cells. As a result, this horizontal transfer triggered the development of therapy-resistant metastases in BC (Sansone et al., 2017a). Furthermore, a recent study revealed that $\mathrm{O}^{2}$-3-Aminopropyl diazeniumdiolates $3 \mathrm{a}-\mathrm{f}$ was created to markedly suppress the metastatic ability of TNBC by attenuating microvesicles formation in an NO-dependent manner (Kang et al., 2018). Welte et al. (2016) highlighted that the mTOR pathway promoted G-Colony-stimulating factors expression in preclinical models of BC which further resulted in immunosuppressive tumor microenvironment. 


\section{ROLES OF EXOSOMES IN BC DRUG RESISTANCE AND RADIORESISTANCE}

The resistance of BC cells to chemotherapy, endocrine therapy, anti-HER-2-targeted therapy, and radiotherapy currently limits their treatment efficacy. As a result, scrutiny of the underlying mechanisms leading to resistance is essential to develop more effective therapies for patients with BC. As exosomes are involved in intercellular communication by transmitting their cargos not only to recipient cells in their immediate surroundings but also to distant organs, it is reasonable to envisage their role in inducing or improving the resistance of BC cells. The contents of exosomes are highly diverse, in which several kinds of RNAs, proteins, DNAs have been detected, including oncoproteins, DNA fragments, mRNAs and non-coding RNAs, such as miRNAs, tRNA, snRNA, longnon-coding RNA (lncRNA), and circular RNA (circRNAs) (Murillo et al., 2019). Mastering our understanding of the underlying resistance mechanisms will aid in enhancing cancer treatments and subsequently improving patient outcomes. The explicit mechanisms by which resistance may exist are outlined in this section.

\section{Drug Resistance}

Recent evidence has revealed that exosomes play a major role not only in regulating drug resistance but also in transferring resistance to drug-sensitive BC cells. Furthermore, resistance can be inherent in the tumor cell itself or initiated by treatment with anticancer drugs (acquired resistance) and results in poor prognosis in cancer patients (Pereira et al., 2020). Clear and definite individual causes underlying chemoresistance could facilitate the development of more appropriate therapies for each patient. In this section, we discuss how transmission of exosomes induces the development of $\mathrm{BC}$ drug resistance and how molecular insights into exosome contents have unraveled their pivotal roles.

\section{Exosomal Non-coding RNAs in Breast Cancer Drug Resistance}

A growing volume of literature during the past decade indicates that non-coding RNAs are not the previously commonly deemed non-functional "junk" (Slack, 2006). Indeed, these RNAs, despite not being involved in encoding proteins, are mysterious regulators of most and appear to exert crucial functions in a wide range of human diseases, notably cancer. They have been identified as both oncogenic drivers and tumor suppressors that influence cancer progression in complex ways (Slack and Chinnaiyan, 2019). Non-coding RNAs are divided broadly by their size. Small non-coding RNAs, such as miRNAs, are capable of regulating mRNAs through binding to complementary sequences, while lncRNAs, including subtypes such as pseudogenes and circRNAs, are essential for creating a flexible molecular scaffold to sustain cellular activities (Anastasiadou et al., 2018). On the meantime, studies focused on investigating the effects of tRNA and its fragment deepened our understanding of $\mathrm{BC}$ malignant process. A tRNA fragment $5^{\prime}$-tiRNA ${ }^{\text {Val }}$ was demonstrated capable of suppressing BC development through inhibiting FZD3/Wnt/B-Catenin signaling pathway, which could serve as a potential diagnostic biomarker (Mo et al., 2019). Although, there do not have adequate evidence demonstrate the relation between tRNA and $\mathrm{BC}$ in the aspect of exosomes, advancements of high-throughput sequencing technology may aid in better identifications of their roles and mechnisms in cancer.

Exosome cargo includes several types of nucleic acids, including miRNAs, lncRNAs and mRNAs. Substantial evidence indicates that exosomes are crucially involved in the development of BC drug resistance by transmitting RNA. For instance, one finding indicated the possibility that drug-resistant $\mathrm{BC}$ cells conferred resistance to drug-sensitive $\mathrm{BC}$ cells partly by releasing specific exosomal miRNAs and thereby enhancing the general resistance of MCF-7 /S after co-culture (Chen et al., 2014). Specifically, the drug resistance trait was induced after transfer of exosomal miR-100, miR-222 and miR-30a from resistant BC cell lines MCF-7/Adr and MCF-7/Doc to MCF-7/S recipient sensitive cells. However, the underlying mechanisms of these effects have yet to be elucidated. Wei et al. (2014) demonstrated that specific exosomal miRNA-221/222 secreted by tamoxifenresistant ER- $\alpha$-positive $B C$ cells reduce expression of P27 and ER$\alpha$ in tamoxifen-sensitive $B C$ cells, resulting in the transfer of drug resistance (Wei et al., 2014). Furthermore, researchers found that extracellular vesicles originating from HCC1806 TNBC cells have the ability to induce proliferation and transfer drug resistance to non-tumorigenic MCF10A breast cells, which is potentially mediated by alterations in gene and miRNA expression correlated with cell proliferation, invasion, and migration (Ozawa et al., 2018). Similarly, a recent study focused on exploring Adriamycin resistance in $\mathrm{BC}$ cells, revealing that exosomal miR-221-3p was the key in promoting resistance by targeting PIK3R1 (Pan et al., 2020). In addition, exosomes may modulate resistance and migration capacity from chemoresistant $\mathrm{BC}$ cells to sensitive cells, partly by the encapsulation and transfer of miR-155, which is also associated with cancer stem cells and the process of epithelial-tomesenchymal transition (Santos et al., 2018). Notably, according to recent studies, various factors or pathways are amended to impair the efficiency of chemotherapeutic agents and induce drug resistance. For example, stimulation of the cancer stem-like cell phenotype is closely related to chemoresistance in tumors by differentiation into different cell types (Das and Law, 2018). Chemotherapy induces BC cells to secrete multiple extracellular vesicle encapsulated miRNAs, including miRNA-9-5p, miRNA195-5p, and miRNA-203a-3p, which led to adaptation of cancer stem-like cell traits by simultaneously targeting the transcription factor One Cut Homeobox 2. In turn, downregulation of these miRNAs or upregulation of One Cut Homeobox 2 expression abolished the cancer stem-like cell-activating effect of extracellular vesicles from chemotherapy-treated BC cells (Shen et al., 2019).

While $\mathrm{BC}$ cell malignant activities may be modulated by lncRNAs, the underlying drug resistance mechanisms within exosomes are still largely unknown. A recent study indicated that the lncRNA AFAP1-AS1 is highly expressed in trastuzumabresistant BC cells. Notably, this specific lncRNA disseminated trastuzumab resistance to other $\mathrm{BC}$ cells through transmission in 
exosomes to further associate with AUF1 and activated ERBB2 translation, which did not influence mRNA expression (Han et al., 2020a). Another lncRNA with abnormal expression was demonstrated in a recent finding. Doxorubicin has been widely recognized as a first-line drug in treating BC (Mann et al., 2020). LncRNA H19 was found to be strikingly overexpressed in doxorubicin-resistant $\mathrm{BC}$ cells and was encapsulated into exosomes to transfer drug resistance into drug-sensitive BC cells. Similarly, downregulation of $\mathrm{H} 19$ reversed the chemoresistance of doxorubicin in sensitive BC cells (Wang et al., 2020). The oncogenic function of lncRNA AGAP2-AS1 was previously characterized in gastric cancer (Qi et al., 2017). However, its underlying functions in $\mathrm{BC}$ remain unknown. A novel study concluded that AGAP2-AS1 is dysregulated in trastuzumabresistant $\mathrm{BC}$ cells and plays critical roles in enhancing trastuzumab resistance by packaging into exosomes in an hnRNPA2B1-dependent manner and inhibiting trastuzumabinduced cell cytotoxicity (Zheng et al., 2019). In addition, a previous finding verified the association between the lncRNA small nucleolar RNA host gene 14 and the response to trastuzumab in BC cells. Functional experimentation and analysis demonstrated that this IncRNA aided in enhancing trastuzumab resistance possibly by modulating the apoptosis-related signaling pathway and can be exploited as a potential diagnostic biomarker in future development (Dong et al., 2018). Another novel study first compared the exosomal lncRNA urothelial carcinomaassociated 1 loading differences between tamoxifen-resistant cells and tamoxifen-sensitive cells in estrogen-positive BC, revealing significantly increased levels of urothelial carcinoma-associated 1 in tamoxifen-resistant cells, especially in exosomes derived from these cells. These results indicate that the transfer of urothelial carcinoma-associated 1 mediated by exosomes might represent a vital mechanism in inducing acquired tamoxifen resistance in BC cells (Xu et al., 2016).

In addition to the various microRNAs and lncRNAs discussed above, circRNAs have also been detected in exosomes. Although exosomal circRNA and its associated functions have been little studied in BC drug resistance, some articles have revealed exosomal circRNA roles in other types of cancers. For example, the metastatic ability of liver cancer cells was enhanced by transferring exosomal circRNA-100338, affecting human umbilical vein endothelial cells and their proangiogenic activity (Huang et al., 2020). Similarly, hepatocellular carcinoma cell metastatic potential was found to be transferred via exosomes with circPTGR1 and its three isoforms (Wang G. et al., 2019). As the liver is the most common site of metastasis in $\mathrm{BC}$, it is plausible to envisage exosomal circRNAs and their effects on $\mathrm{BC}$ progression, and further explorations are warranted in future research.

\section{Exosomal Proteins in Breast Cancer Drug Resistance}

Various oncogenic proteins and oncometabolites are prominent players in BC progression. Data provided by Mishra et al. (2018) supported that ADHFE1 which is a BC oncogene protein contributed to the accumulation of oncometabolite 2-hydroxyglutarate and induced metabolic reprogramming through MYC signaling. In addition to nucleic acids, protein cargo in exosomes is also involved in the drug resistance of BC. TrpC5, a potential transient receptor protein, prompted the formation of the multidrug efflux transporter P-glycoprotein via a $\mathrm{Ca}^{2}+$ and activated T-cell isoform c3-induced mechanism through accumulation and transfer in extracellular vesicles. In addition, upregulation of exosomal Trpc5 has a unique role in trapping Adriamycin and partially transferring chemoresistance from drug-resistant BC cells to non-resistant cells (Ma et al., 2014). Exosomal delivery of P-gp, a transmembrane protein with a higher concentration in drug-resistant BC cells, caused efflux of drug substrates to maintain sublethal intracellular drug levels. It was suggested to play an important role in transferring drug resistance from docetaxel-resistant cells to drug-sensitive cells in BC (Lv et al., 2014). In addition, a novel study demonstrated that UCH-L1-containing exosomes secreted by Adriamycinresistant human $\mathrm{BC}$ cells were taken up by Adriamycin-sensitive human BC cells in a time-dependent manner and ultimately contributed to the chemoresistance phenotype. This individual trait also entailed potential diagnostic implications for UCHL1 (Ning et al., 2017). Similarly, Yang et al. (2017) found that glutathione S-transferase P1-containing exosomes have the ability to transfer drug resistance from Adriamycin-resistant $\mathrm{BC}$ cells to Adriamycin-sensitive $\mathrm{BC}$ cells by the translocation of glutathione S-transferase P1, which is a metabolic enzyme that reportedly decreases the efficiency of several anticancer drugs via conjugation with glutathione (Coles and Kadlubar, 2003). Expression of this transferase was considerably higher in Adriamycin-resistant BC cells and their corresponding exosomes. More importantly, increasing the concentration of this enzyme in circulating exosomes should be explored for clinical prediction applications (Yang et al., 2017).

Unfortunately, chemotherapy stimulates the creation of undesirable products while killing cancer cells. It was demonstrated that intensive chemoresistant therapeutic-induced senescent cells were generated after chemotherapy and helped form stem cell niches that contributed to metastasis. Importantly, increased extracellular vesicles and exosomes containing key proteins involved in tumor progression were harvested from and regulated by therapeutically induced senescent cells in TNBC cells. Additionally, expression of the multidrug resistance protein $1 /$ p-glycoprotein was increased in therapeutically induced senescent cells. Taken together, these finding indicate that extracellular vesicles in therapeutically induced senescent cells TNBC cells are overexpressed and partially aid in conveying resistance to chemotherapy (Kavanagh et al., 2017).

\section{Exosomes Involved in Other Mechanisms of Drug Resistance in Breast Cancer}

Although various exosomal cargos are closely correlated with BC therapeutic resistance, exosomes themselves are also influenced and play vital roles in impairing BC therapy efficiency. For example, a previous investigation found that exosomes influenced the outcomes of antibody drugs by regulating their binding to tumor cells (Aung et al., 2011). Besides, exosomes released from HER2-overexpressing BC cells containing the HER2 protein neutralized the HER2-targeted antibody-based drug trastuzumab and attenuated its interaction with the 
intended BC target cells, leading to enhanced aggressiveness (Ciravolo et al., 2012). Intriguingly, exosomes can also function as novel shelters. It was revealed that the epidermal growth factor receptor, which is essential in promoting $\mathrm{BC}$ progression, was protected in exosomal compartments, allowing it to evade attacks from epidermal growth factor receptor small inhibitors in a TNBC model. As a result, drug resistance was triggered, and related signaling pathways were stimulated to activate proliferation and migration in TNBC recipient cells (Hung et al., 2019).

\section{Resistance to Radiotherapy}

Radiotherapy also provides an effective option for treating $\mathrm{BC}$; however, resistance to radiotherapy has been identified in large-scale studies. Some findings indicated that exosomes engaged in this process. For example, a study investigating the effect of X-ray irradiation on differences in exosome activity in MCF-7 BC cells found that exosome biogenesis and secretion were activated in a dose-dependent manner, revealing the potential of exosomes to convey resistance to radiotherapy in BC cells (Jabbari et al., 2019). In addition, cargo from irradiated cell-derived exosomes was distinct from that of non-irradiated cells, indicating alterations in the exosomal formation system (Mutschelknaus et al., 2017). However, one study demonstrated that irradiated mouse BC cells secreted exosomes that transferred double-stranded DNA to dendritic cells and stimulated dendritic cell activation of IFN-I, which was vital for recruiting dendritic cells and indicated promising prospects for connecting radiotherapy with immunotherapy in BC. Specifically, exosomes derived from irradiated cells elicited immune responses of tumor-specific CD8 + T-cells and inhibition of tumor size in a mouse BC model (Diamond et al., 2018). These data showed that BC-derived exosomes mediate radioresistance by transferring adverse molecules from irradiated cells toward target cells. However, further investigations are warranted to reveal the underlying roles of BC-derived exosomes in the generation of resistance. Under these circumstances, it is a promising step to develop additional treatment approaches following radiotherapy by interfering with exosomal biogenesis and function.

\section{Exosomes in Relieving or Reversing Therapy Resistance of Breast Cancer}

While many drug-resistance incidents induced by exosomes and their contents in BC cells have been discussed above, it is noteworthy that some findings also revealed that exosomes have a role in relieving or reversing resistance in BC. A novel study identified an agent called $d$ rhamnose $\beta$-hederin that attenuates resistance traits in docetaxel-resistant $\mathrm{BC}$ cells and reduces tumor burden by decreasing exosome secretion (Chen et al., 2018). In addition, exosomes secreted from multidrug-resistant BC cells were inhibited by psoralen and impaired resistance via the PPAR and p53 signaling pathways. This report also provided novel insight into treating BC resistance to chemotherapy (Wang et al., 2016). In addition, blocking PGE2/EP4 signaling contributed to $\beta$-catenin removal from $\mathrm{BC}$ stem cells through EVs/exosomes.
As a result, the number of chemotherapy-resistant cancer stem cells was reduced, which enhanced chemosensitivity in BC cells (Lin et al., 2018). It was also demonstrated that miRNA-770 can be transferred by exosomes from TNBC cells to tumor-associated macrophages, which sensitize responses to chemotherapy by regulating macrophage polarization ( $\mathrm{Li} \mathrm{Y}$. et al., 2018). Furthermore, trastuzumab chemoresistance was reversed after transmitting exosomal miRNA-567 into BC cells and suppressing autophagy, which is important in maintaining the survival of cancer cells under various adverse conditions, including nutrient deficiency, chemotherapy, and radiotherapy (Han et al., 2020b).

These data indicated that $\mathrm{BC}$ exosomes mediate resistance via multiple different interventions and revealed conceivable values of exosomes as diagnostic and prognostic biomarkers. Nevertheless, clinical trials are needed to further demonstrate the ability to target exosomes and their effects in practical treatment.

\section{THERAPEUTIC APPLICATIONS OF EXOSOMES IN BREAST CANCER}

Although developing strategies have been applied to treat BC, including surgical intervention, chemotherapy, radiotherapy, and hormone therapy, challenges have also arisen (Harbeck and Gnant, 2017). Drug resistance, high toxicity and other mechanisms were found to be responsible for treatment failure. To tackle these obstacles, more attention should be paid to identifying novel therapies and delivering technologies for BC. According to this trend, precision oncology has aroused attention of researchers as their potential capability in discovering predicting molecular biomarkers. Currently, exosomes have gained interest as a novel type of cancer vaccine and potential targeted drug delivery vehicle because they significantly inhibit BC progression without obvious toxicity or immunogenicity (Gong et al., 2019). In this section, we discuss the pleiotropic functions of exosomes in BC therapeutics and their novel roles in advanced BC are also described (Table 1).

\section{Exosomes as Complementary Tools in Liquid Biopsy}

In contrast to traditional "solid biopsies," liquid biopsy provides a non-invasive method to both consistently monitoring and improving our knowledge about the BC metastatic process (Nadal et al., 2012). By analyzing immunosuppressive profiles in liquid biopsy, researchers revealed that elevated level of eMDSCs exacerbated immunosuppression which facilitated identification of poor neoadjuvant chemotherapy BC responders (SalvadorColoma et al., 2020). Compared with other types of liquid biopsies, exosomes have advantages in different aspects. First, the cup-shaped appearance makes it easier to distinguish exosomes by using electron microscopy in comparison to other subcellular particles such as apoptotic bodies and microvesicles (Luga et al., 2012). Second, differ from other new biomarker sources, mature technologies have been developed to isolate and characterize exosomes (Lobb et al., 2015). Third, exosomes are stable in the body circulation and are easy detected in almost every 
potential body fluid. Last but not least, they can also mirror their underlying origin cell markers through presenting specific surface proteins (Chavez-Muñoz et al., 2008). Consequently, exosomes extracted from human body fluid are under extensive investigation to further allow for better treatment choices. For example, fifty-three localized BC patients received neoadjuvant therapy, after analyzing exosomal miRNAs and circulating tumor cells as potential biomarkers, researchers found that higher expression of exosomal miRNA-21, miRNA-222, and miRNA-155 were strongly correlated with different diagnosis and treatment response in diverse patients (Rodríguez-Martínez et al., 2019). In addition, a set of exosomal miRNAs in circulating system targeting immune maturation related pathway, predicted poor response to neoadjuvant therapy preceded to chemotherapy in TNBC (Salvador-Coloma et al., 2020). Besides, another investigation showed that the level of lncRNA-HOTAIR in circulatory exosome was positively associated with the status of HER2 in BC tissues (Wang Y. L. et al., 2019).

Not only exosomes, other extracellular vesicles are found to play a role in liquid biopsy. With clinical validation, a novel technology using nanopatterned microchips was reported to monitor tumor progression and metastasis. This technology demonstrated matrix metalloproteases-14 on extracellular vesicles to detect cell invasiveness in vitro (Zhang et al., 2020). However, an investigation into the comparison between extracellular DNA and circulating tumor DNA found that while copy number variants were both detectable in two kinds of DNA, circulating tumor DNA have better sensitivity in serial monitor of BC (Ruhen et al., 2020).

To sum up, different content of bioliquid exosomes may applied in diagnosing and predicting treatment response of patients with BC.

\section{Exosomes as Potential Carriers}

Exosomes are promising natural carriers of biomolecules or anti-tumor agents because they decrease clearance in the circular system and increase specificity toward target cells after modification by surface proteins and engineered targeting methods. For example, bone marrow-derived mesenchymal stem cell-released exosomes were designed to be loaded with antimiRNA drugs and efficiently transferred into BC cells in vitro. Delivery of anti-miRNA-142-3p downregulated levels of miRNA142-3p, which further increased the transcription of certain target genes and consequently led to reduced tumorigenicity (Naseri et al., 2018). In addition, exosomes can be used as novel carriers to strengthen BC treatment efficiency after loading with specific agents and subsequent uptake by target cells. For instance, M1-polarized macrophages secrete exosomes that are used as paclitaxel carriers with a specific nanoformulation that induces a pro-inflammatory environment. After coincubation with the complex, expression of caspase-3 was increased in 4T1 BC cells in mice, which indicated the role of exosomes in enhancing the antitumor effects of chemotherapeutics (Wang P. et al., 2019). One of the advantages of exosomes is their low toxicity; doxorubicinloaded exosomes were less toxic than pure doxorubicin but did not exhibit decreased drug efficacy as demonstrated both in vitro and in vivo (Toffoli et al., 2015).
Exosomes were also engineered to retarget recipient cells to increase the efficiency of delivery and improve anti-tumor ability. TNBC has a poor prognosis due to a lack of appropriate therapeutic targets, and clinical chemotherapeutics are often restricted by easy drug removal. As a result, exosomes that possess traits of delivering cargos without elimination in circular systems were designed to improve the efficacy of chemotherapeutics. In a recent study, macrophages released exosomes with coated nanoparticles engineered by a peptide that was capable of specifically targeting the mesenchymal-epithelial transition factor. As this transition factor was found to be overexpressed in TNBC cells, the cellular absorption efficiency was greatly improved and further led to enhanced inhibition of tumor growth and increased tumor apoptosis (Li et al., 2020). In addition, another study demonstrated that modifying exosomes with unique monoclonal antibodies aided in improving targeted BC immunotherapy. Mechanistically, synthetic multivalent antibody redirected exosomes were developed to both target CD3 $\mathrm{T}$ cells and BC-associated HER2 receptors. This dual targeting induced specific anti-tumor activity as cytotoxic $\mathrm{T}$ cells were activated to attack HER2-expressing BC cells (Shi et al., 2020). In previous reports, exosomes were directly engineered to target BC cells. However, exosomes can be engineered and retargeted indirectly by moderating their parental cells. Consequently, let7a miRNA was enclosed in exosomes and delivered to EGFRexpressing xenograft $\mathrm{BC}$ tissues after consistent intravenous injection (Ohno et al., 2013; Table 2).

\section{Exosome-Based Vaccines}

Owing to tumor evasion of the immune system, stimulating immune responses using an exosome-based vaccine represents a promising treatment for cancer. Li R. et al. (2018) developed a novel tumor vaccine that combined HER2-specific dendritic cell-released exosomes with polyclonal CD4 $+\mathrm{T}$ cells. This complex demonstrated efficacy against HER2-positive tumors in an HER2 antibody therapy-resistant mouse model (Li R. et al., 2018). Therefore, this novel vaccine will likely aid in treating HER2-positive BC patients. In addition, as tumorderived exosomes contain various components, including tumorspecific and associated antigens, it is reasonable to propose the hypothesis that tumor-derived exosomes can be used as sources to stimulate immunity against tumor cells. It was demonstrated that after exosomes were modified with miR155, miR-142, and let-7i, the immune stimulatory ability was enhanced, leading to the maturation of dendritic cells (Taghikhani et al., 2019; Table 2).

\section{Implications of Exosomes in Metastatic Breast Cancer Therapy}

Exosomes are thought to involved in multiple stages during invasive processes and likely contributed to early steps participated in metastasis. For example, it was collaborated that ASPH network regulated designated exosomes to enable deliver of pro-oncogenic secretome and disseminating for long-distance matastasis (Lin et al., 2019). Besides, exosome-mediated deliever of tumor-secreted miR-105 was revealed selectively destroyed 
TABLE 2 | The potential roles of exosomes in treating BC.

\begin{tabular}{|c|c|c|c|c|c|}
\hline Roles & Donor cells & Specific drug & Recipient cells & Results & References \\
\hline \multirow{3}{*}{$\begin{array}{l}\text { Exosomes as } \\
\text { complementary tools in } \\
\text { liquid biopsy }\end{array}$} & l & l & / & Predicting outcomes & $\begin{array}{l}\text { Rodríguez-Martínez } \\
\text { et al., } 2019\end{array}$ \\
\hline & & & & Predicting resistance & $\begin{array}{l}\text { Salvador-Coloma } \\
\text { et al., } 2020\end{array}$ \\
\hline & & & & Aiding treatment choices & $\begin{array}{l}\text { Wang Y. L. et al., } \\
2019\end{array}$ \\
\hline \multirow[t]{4}{*}{$\begin{array}{l}\text { Exosomes as potential } \\
\text { carriers }\end{array}$} & $\begin{array}{l}\text { Bone marrow-derived } \\
\text { mesenchymal stem cells }\end{array}$ & Anti-miRNA-142-3p & BC cells & $\begin{array}{l}\text { The tumorigenicity of } \mathrm{BC} \text { was } \\
\text { reduced }\end{array}$ & Naseri et al., 2018 \\
\hline & $\begin{array}{l}\text { M1-polarized } \\
\text { macrophages }\end{array}$ & Paclitaxel & $\mathrm{BC}$ cells & $\begin{array}{l}\text { Paclitaxel-M1-exosomes } \\
\text { exhibited higher anti-tumor effects }\end{array}$ & Wang P. et al., 2019 \\
\hline & Macrophages & Doxorubicin & TNBC cells & $\begin{array}{l}\text { Increased inhibition of tumor } \\
\text { growth and induced intense } \\
\text { tumor apoptosis }\end{array}$ & Li et al., 2020 \\
\hline & HEK293T cells & $\begin{array}{l}\text { Anti-human CD3 and } \\
\text { anti-human HER2 } \\
\text { antibodies }\end{array}$ & HER2-expressing BC cells & $\begin{array}{l}\text { Exhibited highly potent and } \\
\text { specific anti-tumor activity }\end{array}$ & Shi et al., 2020 \\
\hline \multirow[t]{2}{*}{$\begin{array}{l}\text { Exosome-based } \\
\text { vaccines }\end{array}$} & $\begin{array}{l}\text { Her2 specific dendritic } \\
\text { cells }\end{array}$ & $\begin{array}{l}\text { Polyclonal CD4 + T } \\
\text { cells }\end{array}$ & HER2-positive BC cells & $\begin{array}{l}\text { CTL proliferation, IFN- } \gamma \\
\text { production, and rescuing CTL } \\
\text { cytotoxic effect was induced }\end{array}$ & Li R. et al., 2018 \\
\hline & BC cells & $\begin{array}{l}\text { MiR-155, miR-142, } \\
\text { and let-7i }\end{array}$ & Immature dendritic cells & $\begin{array}{l}\text { The immune stimulation ability } \\
\text { was enhanced and led to } \\
\text { maturation of dendritic cells }\end{array}$ & $\begin{array}{l}\text { Taghikhani et al., } \\
2019\end{array}$ \\
\hline \multirow[t]{4}{*}{$\begin{array}{l}\text { Implications of exosomes } \\
\text { in metastatic BC therapy }\end{array}$} & Nischarin-positive cells & Nischarin & BC cells & $\begin{array}{l}\text { BC cells motility and tumor } \\
\text { growth were reduced }\end{array}$ & Maziveyi et al., 2019 \\
\hline & $\mathrm{BC}$ cells & $\begin{array}{l}\text { CBSA/ } \\
\text { siS100A4@Exosome }\end{array}$ & Lung pre-metastatic niche & $\begin{array}{l}\text { Postoperative breast cancer } \\
\text { metastasis was suppressed }\end{array}$ & Zhao et al., 2020 \\
\hline & $\mathrm{BC}$ cells & $\begin{array}{l}\text { Antibody-tagged } \\
\text { exosomes }\end{array}$ & Macrophages & $\begin{array}{l}\text { EV-triggered metastasis in } \mathrm{BC} \\
\text { was disrupted }\end{array}$ & $\begin{array}{l}\text { Nishida-Aoki et al., } \\
2017\end{array}$ \\
\hline & BC cells & MiRNA-126 & A546 lung cancer cells & $\begin{array}{l}\text { The formulation of lung } \\
\text { metastasis was inhibited }\end{array}$ & Nie et al., 2020 \\
\hline \multirow[t]{2}{*}{$\begin{array}{l}\text { Modulation of exosomal } \\
\text { secretion }\end{array}$} & $\begin{array}{l}\text { Docetaxel-resistant BC } \\
\text { cells }\end{array}$ & d Rhamnose $\beta$-hederin & / & $\begin{array}{l}\text { The formation and release of } \\
\text { exosomes derived from } \\
\text { docetaxel-resistant } \mathrm{BC} \text { cells was } \\
\text { reduced }\end{array}$ & Chen et al., 2018 \\
\hline & $\mathrm{BC}$ cells & $\begin{array}{l}\text { A selective leptin } \\
\text { receptor } \\
\text { antagonist-the peptide } \\
\text { LDFI (Leu-Asp-Phe-lle) }\end{array}$ & & $\begin{array}{l}\text { The release of } \mathrm{BC} \text { cell-derived } \\
\text { exosomes was impaired }\end{array}$ & Giordano et al., 2019 \\
\hline
\end{tabular}

tight junctions and the integrity of natural barriers which led to enhanced metastasis in BC (Zhou et al., 2014). At the same time, abundant studies also revealed exosomes exerted influence on reducing metastasis activity in BC. Sulfisoxazole is an oral antibiotic that functions as an anti-tumor and antimetastatic drug in BC xenografts. Further study using pharmacological and genetic methods and a direct binding assay demonstrated that sulfisoxazole interfered with endothelin receptor A and decreased the release of exosomes, which ultimately inhibited progression and metastasis in BC cells (Im et al., 2019). Additionally, nischarin, a tumor suppressor, was previously found to regulate early metastatic events in BC (Maziveyi et al., 2018). In further research, Maziveyi et al. (2019) revealed a novel role for nischarin in preventing BC cell motility and tumor growth by regulating Rab14 activity and consequently secreting exosomes capable of controlling tumor malignancy. In addition, a recent study suggested that antisense non-coding mitochondrial RNA was a novel target for cancer therapy in BC. After treatment with an antisense oligonucleotide, levels of antisense non-coding mitochondrial RNA were decreased in malignant BC cells. As a result, exosomes released from these $\mathrm{BC}$ cells were capable of reducing tumorigenic properties in recipient cells, which shed light on the positive relationship between the transfer of exosomes derived from antisense non-coding mitochondrial RNA knockdown cells and inhibition of the development of BC metastatic niches (Lobos-González et al., 2020). Similarly, researchers found that exosome-mediated siRNA presented excellent biocompatibility and high affinity toward the lung, indicating a promising strategy to inhibit metastasis in postoperative BC patients (Zhao et al., 2020). In addition, a novel therapeutic strategy exploited antibodies to target exosomes, and efficiency was confirmed both in vivo and in vitro, as antibody-tagged cancer-derived exosomes were internalized and eliminated by macrophages, leading to decreased metastatic incidence (Nishida-Aoki et al., 2017). Interestingly, specific cancer cell-released exosomes also exerted their function in treating other cancer cells. For example, exosomes released from BC cells were found in a recent study to inhibit lung cancer cell proliferation and migration as they possessed organotropic properties. Specifically, exosomes were exploited as miRNA-126 protective nanocarriers and taken up by A546 lung cancer cells, which inhibited the PTEN/PI3K/AKT signaling pathway 
and suppressed pulmonary tumor cell metastatic ability (Nie et al., 2020). The above findings discussed the potential role of exosomes in aiding advanced metastatic BC treatments, which give us a broaden horizon over selecting regimes (Table 2).

\section{Modulation of Exosomal Secretion}

As discussed in previous studies, modulated exosomal secretion is also involved in improving BC clinical treatment. For example, a specific drug called $\mathrm{d}$ Rhamnose $\beta$-hederin was investigated to explore its effects in treating BC. It was demonstrated that this novel drug reduced the formation and release of exosomes derived from docetaxel-resistant BC cells, which contributed to reversing docetaxel resistance (Chen et al., 2018). Sulfisoxazole, an oral antibiotic, was found to be important in effectively suppressing BC metastasis and tumor cell growth by inhibiting the biogenesis and secretion of small extracellular vesicles (Im et al., 2019). Additionally, the obesity hormone leptin was found to be capable of regulating the biogenesis and generation of exosomes in both MCF-7 and MDA-MB-231 $\mathrm{BC}$ cells, representing a potential strategy to interrupt harmful intercommunication between cells (Giordano et al., 2019).

While exosomes offer solutions to many challenges that are encountered by clinicians in the treatment of BC, they are not devoid of obstacles. Many mechanisms inhibit their successful therapeutic application, including potential immunogenicity, the risk of stimulating metastasis, and intracellular heterogeneity (Pullan et al., 2019). With the previously published positive results, clinical trials of exosome-based delivery methods and deeper investigations are needed to confirm their clinical applicability in solid tumors (Table 2).

\section{CONCLUSION AND FUTURE PERSPECTIVES}

Exosomes are a range of nanosized extracellular vesicles that play pleiotropic roles in $\mathrm{BC}$ development and progression. By transferring various cargos, including proteins, lipids and RNA, they are capable of modulating intercellular interplay in the

\section{REFERENCES}

Anastasiadou, E., Jacob, L. S., and Slack, F. J. (2018). Non-coding RNA networks in cancer. Nat. Rev. Cancer 18, 5-18.

Aung, T., Chapuy, B., Vogel, D., Wenzel, D., Oppermann, M., Lahmann, M., et al. (2011). Exosomal evasion of humoral immunotherapy in aggressive B-cell lymphoma modulated by ATP-binding cassette transporter A3. Proc. Natl. Acad. Sci. U.S.A. 108, 15336-15341. doi: 10.1073/pnas.110285 5108

Baroni, S., Romero-Cordoba, S., Plantamura, I., Dugo, M., D’Ippolito, E., Cataldo, A., et al. (2016). Exosome-mediated delivery of miR-9 induces cancer-associated fibroblast-like properties in human breast fibroblasts. Cell Death Dis. 7:e2312. doi: $10.1038 /$ cddis.2016.224

Bissell, M. J., and Hines, W. C. (2011). Why don't we get more cancer? A proposed role of the microenvironment in restraining cancer progression. Nat. Med. 17, 320-329. doi: 10.1038/nm.2328

Boyle, S. T., Poltavets, V., Kular, J., Pyne, N. T., Sandow, J. J., Lewis, A. C., et al. (2020). ROCK-mediated selective activation of PERK signalling causes tumor microenvironment. This review brings together their roles in $\mathrm{BC}$ therapy resistance and their mediating and communicating functions in the tumor microenvironment.

Exosomes may represent novel and accessible biomarkers of various cancers, as they are nearly ubiquitous in human cells. Moreover, there is rapidly growing evidence that exosomes are potential carriers of therapeutic agents or can be designed as vaccines to improve targeting and treatment efficiency in BC cells. Thus, the role of exosomes and their contents has become a promising research hotspot. However, which method is optimal for utilizing engineered exosomes is still unclear, and more efforts are warranted for safer and better clinical application of exosomes. Taken together, we summarize the pivotal roles of exosomes in orchestrating the tumor microenvironment and promoting BC pathological states. Fresh insights emphasizing treating $\mathrm{BC}$ with exosome-involved strategies are provided as well.

\section{AUTHOR CONTRIBUTIONS}

XW: conceptualization and writing-original draft preparation. CS: writing-review and editing. $\mathrm{XH}$ : visualization. JL and ZF: supervision. WL: project administration. YY: funding acquisition. All authors read and agreed to the published version of the manuscript.

\section{FUNDING}

This work was funded by the National Key Research and Development Program of China (ZDZX2017ZL-01), HighLevel Innovation Team of Nanjing Medical University (JX102GSP201727), Wu Jieping Foundation (320.6750.17006), Key Medical Talents (ZDRCA2016023), 333 Project of Jiangsu Province (BRA2017534 and BRA2015470), The Collaborative Innovation Center for Tumor Individualization Focuses on Open Topics (JX21817902/008), and Project of China Key Research and Development Program Precision Medicine Research (2016YFC0905901).

fibroblast reprogramming and tumour progression through a CRELD2dependent. Nat. Cell Biol. 22, 882-895. doi: 10.1038/s41556-020-0523-y

Bray, F., Ferlay, J., Soerjomataram, I., Siegel, R. L., Torre, L. A., and Jemal, A. (2018). Global cancer statistics 2018: GLOBOCAN estimates of incidence and mortality worldwide for 36 cancers in 185 countries. CA Cancer J. Clin. 68, 394-424. doi: 10.3322/caac. 21492

Caplan, A. I. (1991). Mesenchymal stem cells. J. Orthop. Res. 9, 641-650.

Chaudhary, P., Gibbs, L. D., Maji, S., Lewis, C. M., Suzuki, S., and Vishwanatha, J. K. (2020). Serum exosomal-annexin A2 is associated with African-American triple-negative breast cancer and promotes angiogenesis. Breast Cancer Res. 22:11.

Chavez-Muñoz, C., Morse, J., Kilani, R., and Ghahary, A. (2008). Primary human keratinocytes externalize stratifin protein via exosomes. J. Cell Biochem. 104, 2165-2173. doi: $10.1002 / j c b .21774$

Chen, F., Chen, J., Yang, L., Liu, J., Zhang, X., Zhang, Y., et al. (2019). Extracellular vesicle-packaged HIF-1alpha-stabilizing lncRNA from tumourassociated macrophages regulates aerobic glycolysis of breast cancer cells. Nat. Cell Biol. 21, 498-510. doi: 10.1038/s41556-019-0299-0 
Chen, W. X., Liu, X. M., Lv, M. M., Chen, L., Zhao, J. H., Zhong, S. L., et al. (2014). Exosomes from drug-resistant breast cancer cells transmit chemoresistance by a horizontal transfer of microRNAs. PLoS One 9:e95240. doi: 10.1371/journal. pone.0095240

Chen, W. X., Xu, L. Y., Qian, Q., He, X., Peng, W. T., Fan, W. Q., et al. (2018). d Rhamnose $\beta$-hederin reverses chemoresistance of breast cancer cells by regulating exosome-mediated resistance transmission. Biosci. Rep. 38:BSR20180110.

Chen, Y., Zeng, C., Zhan, Y., Wang, H., Jiang, X., and Li, W. (2017a). Aberrant low expression of $\mathrm{p} 85 \alpha$ in stromal fibroblasts promotes breast cancer cell metastasis through exosome-mediated paracrine Wnt10b. Oncogene 36, 4692-4705. doi: 10.1038/onc. 2017.100

Chen, Y., Zhang, S., Wang, Q., and Zhang, X. (2017b). Tumor-recruited M2 macrophages promote gastric and breast cancer metastasis via M2 macrophagesecreted CHI3L1 protein. J. Hematol. Oncol. 10:36.

Choi, J., Cha, Y. J., and Koo, J. S. (2018). Adipocyte biology in breast cancer: from silent bystander to active facilitator. Prog. Lipid Res. 69, 11-20. doi: 10.1016/j.plipres.2017.11.002

Ciravolo, V., Huber, V., Ghedini, G. C., Venturelli, E., Bianchi, F., Campiglio, M., et al. (2012). Potential role of HER2-overexpressing exosomes in countering trastuzumab-based therapy. J. Cell Physiol. 227, 658-667. doi: 10.1002/jcp. 22773

Cocucci, E., and Meldolesi, J. (2015). Ectosomes and exosomes: shedding the confusion between extracellular vesicles. Trends Cell Biol. 25, 364-372. doi: 10.1016/j.tcb.2015.01.004

Coles, B. F., and Kadlubar, F. F. (2003). Detoxification of electrophilic compounds by glutathione S-transferase catalysis: determinants of individual response to chemical carcinogens and chemotherapeutic drugs? Biofactors 17, 115-130. doi: 10.1002/biof.5520170112

Costa, A., Kieffer, Y., Scholer-Dahirel, A., Pelon, F., Bourachot, B., Cardon, M., et al. (2018). Fibroblast heterogeneity and immunosuppressive environment in human breast cancer. Cancer Cell 33, 463-479.e10.

Das, M., and Law, S. (2018). Role of tumor microenvironment in cancer stem cell chemoresistance and recurrence. Int. J. Biochem. Cell Biol. 103, 115-124. doi: 10.1016/j.biocel.2018.08.011

Diamond, J. M., Vanpouille-Box, C., Spada, S., Rudqvist, N. P., Chapman, J. R., Ueberheide, B. M., et al. (2018). Exosomes shuttle TREX1-sensitive IFNstimulatory dsDNA from irradiated cancer cells to DCs. Cancer Immunol. Res. 6, 910-920. doi: 10.1158/2326-6066.cir-17-0581

Diaz Bessone, M. I., Gattas, M. J., Laporte, T., Tanaka, M., and Simian, M. (2019). The Tumor Microenvironment as a regulator of endocrine resistance in breast cancer. Front. Endocrinol. (Lausanne) 10:547. doi: 10.3389/fendo.2019.00547

Dong, H., Wang, W., Chen, R., Zhang, Y., Zou, K., Ye, M., et al. (2018). Exosomemediated transfer of IncRNASNHG14 promotes trastuzumab chemoresistance in breast cancer. Int. J. Oncol. 53, 1013-1026.

Erin, N., Grahovac, J., Brozovic, A., and Efferth, T. (2020). Tumor microenvironment and epithelial mesenchymal transition as targets to overcome tumor multidrug resistance. Drug Resist. Updat 53:100715. doi: 10.1016/j.drup.2020.100715

Feng, D., Zhao, W. L., Ye, Y. Y., Bai, X. C., Liu, R. Q., Chang, L. F., et al. (2010). Cellular internalization of exosomes occurs through phagocytosis. Traffic 11, 675-687.

Gagliano, T., Shah, K., Gargani, S., Lao, L., Alsaleem, M., Chen, J., et al. (2020). PIK3C $\delta$ expression by fibroblasts promotes triple-negative breast cancer progression. J. Clin. Invest. 130, 3188-3204.

Gernapudi, R., Yao, Y., Zhang, Y., Wolfson, B., Roy, S., Duru, N., et al. (2015). Targeting exosomes from preadipocytes inhibits preadipocyte to cancer stem cell signaling in early-stage breast cancer. Breast Cancer Res. Treat. 150, 685695. doi: 10.1007/s10549-015-3326-2

Giordano, C., Gelsomino, L., Barone, I., Panza, S., Augimeri, G., Bonofiglio, D., et al. (2019). Leptin modulates exosome biogenesis in breast cancer cells: an additional mechanism in cell-to-cell communication. J. Clin. Med. 8:107.

Gong, C., Tian, J., Wang, Z., Gao, Y., Wu, X., Ding, X., et al. (2019). Functional exosome-mediated co-delivery of doxorubicin and hydrophobically modified microRNA 159 for triple-negative breast cancer therapy. J. Nanobiotechnol. 17:93.

Ham, S., Lima, L. G., Chai, E. P. Z., Muller, A., Lobb, R. J., Krumeich, S., et al. (2018). Breast cancer-derived exosomes alter macrophage polarization via gp130/STAT3 signaling. Front. Immunol. 9:871. doi: 10.3389/fimmu.2018. 00871

Han, M., Gu, Y., Lu, P., Li, J., Cao, H., Li, X., et al. (2020a). Exosome-mediated lncRNA AFAP1-AS1 promotes trastuzumab resistance through binding with AUF1 and activating ERBB2 translation. Mol. Cancer 19:26.

Han, M., Hu, J., Lu, P., Cao, H., Yu, C., Li, X., et al. (2020b). Exosome-transmitted miR-567 reverses trastuzumab resistance by inhibiting ATG5 in breast cancer. Cell Death Dis. 11:43.

Hannafon, B. N., Carpenter, K. J., Berry, W. L., Janknecht, R., Dooley, W. C., and Ding, W. Q. (2015). Exosome-mediated microRNA signaling from breast cancer cells is altered by the anti-angiogenesis agent docosahexaenoic acid (DHA). Mol. Cancer 14:133.

Harbeck, N., and Gnant, M. (2017). Breast cancer. Lancet 389, 1134-1150.

Huang, X. Y., Huang, Z. L., Huang, J., Xu, B., Huang, X. Y., Xu, Y. H., et al. (2020). Exosomal circRNA-100338 promotes hepatocellular carcinoma metastasis via enhancing invasiveness and angiogenesis. J. Exp. Clin. Cancer Res. 39:20.

Hung, Y., Wang, Y. L., Lin, Y. Z., Chiang, S. F., Wu, W. R., and Wang, S. C. (2019). The exosomal compartment protects epidermal growth factor receptor from small molecule inhibitors. Biochem. Biophys. Res. Commun. 510, 42-47. doi: 10.1016/j.bbrc.2018.12.187

Im, E. J., Lee, C. H., Moon, P. G., Rangaswamy, G. G., Lee, B., Lee, J. M., et al. (2019). Sulfisoxazole inhibits the secretion of small extracellular vesicles by targeting the endothelin receptor A. Nat. Commun. 10:1387.

Jabbari, N., Nawaz, M., and Rezaie, J. (2019). Ionizing radiation increases the activity of exosomal secretory pathway in MCF-7 human breast cancer cells: a possible way to communicate resistance against radiotherapy. Int. J. Mol. Sci. 20:3649. doi: 10.3390/ijms20153649

Jarosz-Biej, M., Smolarczyk, R., Cichoń, T., and Kułach, N. (2019). Tumor microenvironment as A "Game Changer" in cancer radiotherapy. Int. J. Mol. Sci. 20:3212. doi: 10.3390/ijms20133212

Jiang, M., Zhang, W., Zhang, R., Liu, P., Ye, Y., Yu, W., et al. (2020). Cancer exosome-derived miR-9 and miR-181a promote the development of early-stage MDSCs via interfering with SOCS3 and PIAS3 respectively in breast cancer. Oncogene 39, 4681-4694. doi: 10.1038/s41388-020-1322-4

Jung, W. H., Yam, N., Chen, C. C., Elawad, K., Hu, B., and Chen, Y. (2020). Force-dependent extracellular matrix remodeling by early-stage cancer cells alters diffusion and induces carcinoma-associated fibroblasts. Biomaterials 234:119756.

Kalluri, R., and LeBleu, V. S. (2020). The biology, function, and biomedical applications of exosomes. Science 367:eaau6977. doi: 10.1126/science.aau6977

Kalluri, R., and Zeisberg, M. (2006). Fibroblasts in cancer. Nat. Rev. Cancer 6, 392-401.

Kang, F., Zhu, J., Wu, J., Lv, T., Xiang, H., Tian, J., et al. (2018). O(2)-3aminopropyl diazeniumdiolates suppress the progression of highly metastatic triple-negative breast cancer by inhibition of microvesicle formation via nitric oxide-based epigenetic regulation. Chem. Sci. 9, 6893-6898. doi: 10.1039/c8sc0 $0167 \mathrm{~g}$

Kavanagh, E. L., Lindsay, S., Halasz, M., Gubbins, L. C., Weiner-Gorzel, K., Guang, M. H. Z., et al. (2017). Protein and chemotherapy profiling of extracellular vesicles harvested from therapeutic induced senescent triple negative breast cancer cells. Oncogenesis 6:e388. doi: 10.1038/oncsis.2017.82

Kim, J. E., Kim, B. G., Jang, Y., Kang, S., Lee, J. H., and Cho, N. H. (2020). The stromal loss of miR-4516 promotes the FOSL1-dependent proliferation and malignancy of triple negative breast cancer. Cancer Lett. 469, 256-265. doi: 10.1016/j.canlet.2019.10.039

Lee, J. K., Park, S. R., Jung, B. K., Jeon, Y. K., Lee, Y. S., Kim, M. K., et al. (2013). Exosomes derived from mesenchymal stem cells suppress angiogenesis by down-regulating VEGF expression in breast cancer cells. PLoS One 8:e84256. doi: 10.1371 /journal.pone.0084256

Li, R., Chibbar, R., and Xiang, J. (2018). Novel EXO-T vaccine using polyclonal CD4(+) T cells armed with HER2-specific exosomes for HER2-positive breast cancer. Onco Targets Ther. 11, 7089-7093. doi: 10.2147/ott.s184898

Li, S., Wu, Y., Ding, F., Yang, J., Li, J., Gao, X., et al. (2020). Engineering macrophage-derived exosomes for targeted chemotherapy of triple-negative breast cancer. Nanoscale 12, 10854-10862.

Li, Y., Liang, Y., Sang, Y., Song, X., Zhang, H., Liu, Y., et al. (2018). MiR-770 suppresses the chemo-resistance and metastasis of triple negative breast cancer via direct targeting of STMN1. Cell Death Dis. 9:14. 
Liang, Y., Song, X., Li, Y., Chen, B., Zhao, W., Wang, L., et al. (2020). LncRNA BCRT1 promotes breast cancer progression by targeting miR-1303/PTBP3 axis. Mol. Cancer 19:85.

Lin, M. C., Chen, S. Y., He, P. L., Herschman, H., and Li, H. J. (2018). PGE2 /EP4 antagonism enhances tumor chemosensitivity by inducing extracellular vesicle-mediated clearance of cancer stem cells. Int. J. Cancer 143, 1440-1455. doi: $10.1002 / \mathrm{ijc} .31523$

Lin, Q., Chen, X., Meng, F., Ogawa, K., Li, M., Song, R., et al. (2019). ASPH-notch axis guided exosomal delivery of prometastatic secretome renders breast cancer multi-organ metastasis. Mol. Cancer 18:156.

Lobb, R. J., Becker, M., Wen, S. W., Wong, C. S., Wiegmans, A. P., Leimgruber, A., et al. (2015). Optimized exosome isolation protocol for cell culture supernatant and human plasma. J. Extracell. Vesicles 4:27031. doi: 10.3402/jev.v4.27031

Lobos-González, L., Bustos, R., Campos, A., Silva, V., Silva, V., Jeldes, E., et al. (2020). Exosomes released upon mitochondrial ASncmtRNA knockdown reduce tumorigenic properties of malignant breast cancer cells. Sci. Rep. 10:343.

Luga, V., Zhang, L., Viloria-Petit, A. M., Ogunjimi, A. A., Inanlou, M. R., Chiu, E., et al. (2012). Exosomes mediate stromal mobilization of autocrine Wnt-PCP signaling in breast cancer cell migration. Cell 151, 1542-1556. doi: 10.1016/j. cell.2012.11.024

Lv, M. M., Zhu, X. Y., Chen, W. X., Zhong, S. L., Hu, Q., Ma, T. F., et al. (2014). Exosomes mediate drug resistance transfer in MCF-7 breast cancer cells and a probable mechanism is delivery of P-glycoprotein. Tumour Biol. 35, 10773-10779. doi: 10.1007/s13277-014-2377-z

Ma, X., Chen, Z., Hua, D., He, D., Wang, L., Zhang, P., et al. (2014). Essential role for TrpC5-containing extracellular vesicles in breast cancer with chemotherapeutic resistance. Proc. Natl. Acad. Sci. U.S.A. 111, 6389-6394. doi: $10.1073 /$ pnas. 1400272111

Maffey, A., Storini, C., Diceglie, C., Martelli, C., Sironi, L., Calzarossa, C., et al. (2017). Mesenchymal stem cells from tumor microenvironment favour breast cancer stem cell proliferation, cancerogenic and metastatic potential, via ionotropic purinergic signalling. Sci. Rep. 7:13162.

Maji, S., Chaudhary, P., Akopova, I., Nguyen, P. M., Hare, R. J., Gryczynski, I., et al. (2017). Exosomal Annexin II promotes angiogenesis and breast cancer metastasis. Mol. Cancer Res. 15, 93-105. doi: 10.1158/1541-7786.mcr-1 6-0163

Mann, J., Yang, N., Montpetit, R., Kirschenman, R., Lemieux, H., and Goping, I. S. (2020). , BAD sensitizes breast cancer cells to docetaxel with increased mitotic arrest and necroptosis. Sci. Rep. 10:355.

Maziveyi, M., Dong, S., Baranwal, S., and Alahari, S. K. (2018). Nischarin regulates focal adhesion and invadopodia formation in breast cancer cells. Mol. Cancer $17: 21$.

Maziveyi, M., Dong, S., Baranwal, S., Mehrnezhad, A., Rathinam, R., Huckaba, T. M., et al. (2019). Exosomes from nischarin-expressing cells reduce breast cancer cell motility and tumor growth. Cancer Res. 79, 2152-2166. doi: 10.1158/ 0008-5472.can-18-0842

Meads, M. B., Gatenby, R. A., and Dalton, W. S. (2009). Environment-mediated drug resistance: a major contributor to minimal residual disease. Nat. Rev. Cancer 9, 665-674. doi: 10.1038/nrc2714

Mishra, P., Tang, W., Putluri, V., Dorsey, T. H., Jin, F., Wang, F., et al. (2018). ADHFE1 is a breast cancer oncogene and induces metabolic reprogramming. J. Clin. Invest. 128, 323-340. doi: 10.1172/jci93815

Mo, D., Jiang, P., Yang, Y., Mao, X., Tan, X., Tang, X., et al. (2019). A tRNA fragment, $5^{\prime}$-tiRNA(Val), suppresses the Wnt/ $\beta$-catenin signaling pathway by targeting FZD3 in breast cancer. Cancer Lett. 457, 60-73. doi: 10.1016/j.canlet. 2019.05.007

Murillo, O. D., Thistlethwaite, W., Rozowsky, J., Subramanian, S. L., Lucero, R., Shah, N., et al. (2019). exRNA atlas analysis reveals distinct extracellular RNA cargo types and their carriers present across human biofluids. Cell 177, 463-477.e15.

Mutschelknaus, L., Azimzadeh, O., Heider, T., Winkler, K., Vetter, M., Kell, R., et al. (2017). Radiation alters the cargo of exosomes released from squamous head and neck cancer cells to promote migration of recipient cells. Sci. Rep. 7:1 2423.

Nabet, B. Y., Qiu, Y., Shabason, J. E., Wu, T. J., Yoon, T., Kim, B. C., et al. (2017). Exosome RNA unshielding couples stromal activation to pattern recognition receptor signaling in cancer. Cell 170, 352-366.e13.
Nadal, R., Fernandez, A., Sanchez-Rovira, P., Salido, M., Rodríguez, M., GarcíaPuche, J. L., et al. (2012). Biomarkers characterization of circulating tumour cells in breast cancer patients. Breast Cancer Res. 14:R71.

Nakase, I., Kobayashi, N. B., Takatani-Nakase, T., and Yoshida, T. (2015). Active macropinocytosis induction by stimulation of epidermal growth factor receptor and oncogenic Ras expression potentiates cellular uptake efficacy of exosomes. Sci. Rep. 5:10300.

Naseri, Z., Oskuee, R. K., Jaafari, M. R., and Forouzandeh Moghadam, M. (2018). Exosome-mediated delivery of functionally active miRNA-142-3p inhibitor reduces tumorigenicity of breast cancer in vitro and in vivo. Int. J. Nanomed. 13, 7727-7747. doi: 10.2147/ijn.s182384

Ni, C., Fang, Q. Q., Chen, W. Z., Jiang, J. X., Jiang, Z., Ye, J., et al. (2020). Breast cancer-derived exosomes transmit lncRNA SNHG16 to induce CD73 $+\gamma \delta 1$ Treg cells. Signal. Transduct. Target Ther. 5:41.

Nie, H., Xie, X., Zhang, D., Zhou, Y., Li, B., Li, F., et al. (2020). Use of lung-specific exosomes for miRNA-126 delivery in non-small cell lung cancer. Nanoscale 12, 877-887. doi: 10.1039/c9nr09011h

Ning, K., Wang, T., Sun, X., Zhang, P., Chen, Y., Jin, J., et al. (2017). UCH-L1containing exosomes mediate chemotherapeutic resistance transfer in breast cancer. J. Surg. Oncol. 115, 932-940. doi: 10.1002/jso.24614

Nishida-Aoki, N., Tominaga, N., Takeshita, F., Sonoda, H., Yoshioka, Y., and Ochiya, T. (2017). Disruption of circulating extracellular vesicles as a novel therapeutic strategy against cancer metastasis. Mol. Ther. 25, 181-191. doi: 10.1016/j.ymthe.2016.10.009

O’Brien, K., Rani, S., Corcoran, C., Wallace, R., Hughes, L., Friel, A. M., et al. (2013). Exosomes from triple-negative breast cancer cells can transfer phenotypic traits representing their cells of origin to secondary cells. Eur. J. Cancer 49, 1845-1859. doi: 10.1016/j.ejca.2013.01.017

Ohno, S., Takanashi, M., Sudo, K., Ueda, S., Ishikawa, A., Matsuyama, N., et al. (2013). Systemically injected exosomes targeted to EGFR deliver antitumor microRNA to breast cancer cells. Mol. Ther. 21, 185-191. doi: 10.1038/mt.201 2.180

Ozawa, P. M. M., Alkhilaiwi, F., Cavalli, I. J., Malheiros, D., de Souza Fonseca Ribeiro, E. M., and Cavalli, L. R. (2018). Extracellular vesicles from triplenegative breast cancer cells promote proliferation and drug resistance in nontumorigenic breast cells. Breast Cancer Res. Treat. 172, 713-723. doi: 10.1007/ s10549-018-4925-5

Pakravan, K., Babashah, S., Sadeghizadeh, M., Mowla, S. J., MossahebiMohammadi, M., Ataei, F., et al. (2017). MicroRNA-100 shuttled by mesenchymal stem cell-derived exosomes suppresses in vitro angiogenesis through modulating the mTOR/HIF-1 $\alpha /$ VEGF signaling axis in breast cancer cells. Cell Oncol. (Dordr.) 40, 457-470. doi: 10.1007/s13402-017-0 335-7

Pan, X., Hong, X., Lai, J., Cheng, L., Cheng, Y., Yao, M., et al. (2020). Exosomal MicroRNA-221-3p confers adriamycin resistance in breast cancer cells by targeting PIK3R1. Front. Oncol. 10:441. doi: 10.3389/fonc.2020.00441

Pereira, P. M. R., Ragupathi, A., Shmuel, S., Mandleywala, K., Viola, N. T., and Lewis, J. S. (2020). HER2-targeted PET imaging and therapy of hyaluronanmasked HER2-overexpressing breast cancer. Mol. Pharm. 17, 327-337. doi: 10.1021/acs.molpharmaceut.9b01091

Petrova, V., Annicchiarico-Petruzzelli, M., Melino, G., and Amelio, I. (2018). The hypoxic tumour microenvironment. Oncogenesis 7:10.

Picon-Ruiz, M., Morata-Tarifa, C., Valle-Goffin, J. J., Friedman, E. R., and Slingerland, J. M. (2017). Obesity and adverse breast cancer risk and outcome: Mechanistic insights and strategies for intervention. CA Cancer J. Clin. 67, 378-397. doi: 10.3322/caac.21405

Plava, J., Cihova, M., Burikova, M., Matuskova, M., Kucerova, L., and Miklikova, S. (2019). Recent advances in understanding tumor stromamediated chemoresistance in breast cancer. Mol. Cancer 18:67.

Pullan, J. E., Confeld, M. I., Osborn, J. K., Kim, J., Sarkar, K., and Mallik, S. (2019). Exosomes as drug carriers for cancer therapy. Mol. Pharm. 16, 1789-1798. doi: 10.1021/acs.molpharmaceut.9b00104

Qi, F., Liu, X., Wu, H., Yu, X., Wei, C., Huang, X., et al. (2017). Long noncoding AGAP2-AS1 is activated by SP1 and promotes cell proliferation and invasion in gastric cancer. J. Hematol. Oncol. 10:48.

Quail, D. F., and Joyce, J. A. (2013). Microenvironmental regulation of tumor progression and metastasis. Nat. Med. 19, 1423-1437. doi: 10.1038/nm.3394 
Rak, J., and Guha, A. (2012). Extracellular vesicles-vehicles that spread cancer genes. Bioessays 34, 489-497. doi: 10.1002/bies.201100169

Rodríguez-Martínez, A., de Miguel-Pérez, D., Ortega, F. G., García-Puche, J. L., Robles-Fernández, I., Exposito, J., et al. (2019). Exosomal miRNA profile as complementary tool in the diagnostic and prediction of treatment response in localized breast cancer under neoadjuvant chemotherapy. Breast Cancer Res. 21:21.

Ruhen, O., Mirzai, B., Clark, M. E., Nguyen, B., Salomon, C., Erber, W., et al. (2020). Comparison of circulating tumour DNA and extracellular vesicle DNA by lowpass whole-genome sequencing reveals molecular drivers of disease in a breast cancer patient. Biomedicines 9:14. doi: 10.3390/biomedicines9010014

Salvador-Coloma, C., Santaballa, A., Sanmartín, E., Calvo, D., García, A., Hervás, D., et al. (2020). Immunosuppressive profiles in liquid biopsy at diagnosis predict response to neoadjuvant chemotherapy in triple-negative breast cancer. Eur. J. Cancer 139, 119-134. doi: 10.1016/j.ejca.2020.08.020

Sansone, P., Berishaj, M., Rajasekhar, V. K., Ceccarelli, C., Chang, Q., Strillacci, A., et al. (2017a). Evolution of cancer stem-like cells in endocrine-resistant metastatic breast cancers is mediated by stromal microvesicles. Cancer Res. 77, 1927-1941.

Sansone, P., Savini, C., Kurelac, I., Chang, Q., Amato, L. B., Strillacci, A., et al. (2017b). Packaging and transfer of mitochondrial DNA via exosomes regulate escape from dormancy in hormonal therapy-resistant breast cancer. Proc. Natl. Acad. Sci. U.S.A. 114, E9066-E9075.

Santos, J. C., Lima, N. D. S., Sarian, L. O., Matheu, A., Ribeiro, M. L., and Derchain, S. F. M. (2018). Exosome-mediated breast cancer chemoresistance via miR-155 transfer. Sci. Rep. 8:829.

Schreiber, R. D., Old, L. J., and Smyth, M. J. (2011). Cancer immunoediting: integrating immunity's roles in cancer suppression and promotion. Science 331, 1565-1570. doi: 10.1126/science.1203486

Shen, M., Dong, C., Ruan, X., Yan, W., Cao, M., Pizzo, D., et al. (2019). Chemotherapy-induced extracellular vesicle miRNAs promote breast cancer stemness by targeting ONECUT2. Cancer Res. 79, 3608-3621. doi: 10.1158/ 0008-5472.can-18-4055

Shi, X., Cheng, Q., Hou, T., Han, M., Smbatyan, G., Lang, J. E., et al. (2020). Genetically engineered cell-derived nanoparticles for targeted breast cancer immunotherapy. Mol. Ther. 28, 536-547. doi: 10.1016/j.ymthe.2019.11.020

Siegel, R. L., Miller, K. D., and Jemal, A. (2020). Cancer statistics, 2020. CA Cancer J. Clin. 70, 7-30.

Slack, F. J. (2006). Regulatory RNAs and the demise of 'junk' DNA. Genome Biol. $7: 328$.

Slack, F. J., and Chinnaiyan, A. M. (2019). The role of non-coding RNAs in oncology. Cell 179, 1033-1055. doi: 10.1016/j.cell.2019.10.017

Taghikhani, A., Hassan, Z. M., Ebrahimi, M., and Moazzeni, S. M. (2019). microRNA modified tumor-derived exosomes as novel tools for maturation of dendritic cells. J. Cell Physiol. 234, 9417-9427. doi: 10.1002/jcp.27626

Théry, C., Witwer, K. W., Aikawa, E., Alcaraz, M. J., Anderson, J. D., Andriantsitohaina, R., et al. (2018). Minimal information for studies of extracellular vesicles 2018 (MISEV2018): a position statement of the international society for extracellular vesicles and update of the MISEV2014 guidelines. J. Extracell. Vesicles 7:1535750.

Toffoli, G., Hadla, M., Corona, G., Caligiuri, I., Palazzolo, S., Semeraro, S., et al. (2015). Exosomal doxorubicin reduces the cardiac toxicity of doxorubicin. Nanomedicine (Lond.) 10, 2963-2971. doi: 10.2217/nnm.15.118

Toor, S. M., Sasidharan Nair, V., Decock, J., and Elkord, E. (2019). Immune checkpoints in the tumor microenvironment. Semin. Cancer Biol. 65, 1-12. doi: 10.1016/j.semcancer.2019.06.021

Treilleux, I., Blay, J. Y., Bendriss-Vermare, N., Ray-Coquard, I., Bachelot, T., Guastalla, J. P., et al. (2004). Dendritic cell infiltration and prognosis of early stage breast cancer. Clin. Cancer Res. 10, 7466-7474. doi: 10.1158/1078-0432. ccr-04-0684

van Niel, G., D'Angelo, G., and Raposo, G. (2018). Shedding light on the cell biology of extracellular vesicles. Nat. Rev. Mol. Cell Biol. 19, 213-228. doi: 10.1038/nrm.2017.125

Walker, N. D., Elias, M., Guiro, K., Bhatia, R., Greco, S. J., Bryan, M., et al. (2019). Exosomes from differentially activated macrophages influence dormancy or resurgence of breast cancer cells within bone marrow stroma. Cell Death Dis. 10:59.
Wang, G., Liu, W., Zou, Y., Wang, G., Deng, Y., Luo, J., et al. (2019). Three isoforms of exosomal circPTGR1 promote hepatocellular carcinoma metastasis via the miR449a-MET pathway. EBioMedicine 40, 432-445. doi: 10.1016/j.ebiom.2018. 12.062

Wang, P., Wang, H., Huang, Q., Peng, C., Yao, L., Chen, H., et al. (2019). Exosomes from M1-polarized macrophages enhance paclitaxel antitumor activity by activating macrophages-mediated inflammation. Theranostics 9, 1714-1727. doi: 10.7150/thno.30716

Wang, S., Su, X., Xu, M., Xiao, X., Li, X., Li, H., et al. (2019). Exosomes secreted by mesenchymal stromal/stem cell-derived adipocytes promote breast cancer cell growth via activation of Hippo signaling pathway. Stem Cell Res. Ther. 10:117.

Wang, X., Pei, X., Guo, G., Qian, X., Dou, D., Zhang, Z., et al. (2020). Exosomemediated transfer of long noncoding RNA H19 induces doxorubicin resistance in breast cancer. J. Cell Physiol. 235, 6896-6904. doi: 10.1002/jcp.29585

Wang, X., Xu, C., Hua, Y., Sun, L., Cheng, K., Jia, Z., et al. (2016). Exosomes play an important role in the process of psoralen reverse multidrug resistance of breast cancer. J. Exp. Clin. Cancer Res. 35:186.

Wang, Y. L., Liu, L. C., Hung, Y., Chen, C. J., Lin, Y. Z., Wu, W. R., et al. (2019). Long non-coding RNA HOTAIR in circulatory exosomes is correlated with ErbB2/HER2 positivity in breast cancer. Breast 46, 64-69. doi: 10.1016/j.breast. 2019.05.003

Wei, Y., Lai, X., Yu, S., Chen, S., Ma, Y., Zhang, Y., et al. (2014). Exosomal miR221/222 enhances tamoxifen resistance in recipient ER-positive breast cancer cells. Breast Cancer Res. Treat. 147, 423-431. doi: 10.1007/s10549-014-3037-0

Welte, T., Kim, I. S., Tian, L., Gao, X., Wang, H., Li, J., et al. (2016). Oncogenic mTOR signalling recruits myeloid-derived suppressor cells to promote tumour initiation. Nat. Cell Biol. 18, 632-644. doi: 10.1038/ncb3355

Wen, S. W., Lima, L. G., Lobb, R. J., Norris, E. L., Hastie, M. L., Krumeich, S., et al. (2019). Breast cancer-derived exosomes reflect the cell-of-origin phenotype. Proteomics 19:e1800180.

Wen, S. W., Sceneay, J., Lima, L. G., Wong, C. S., Becker, M., Krumeich, S., et al. (2016). The biodistribution and immune suppressive effects of breast cancerderived exosomes. Cancer Res. 76, 6816-6827. doi: 10.1158/0008-5472.can-160868

Wu, H. J., Hao, M., Yeo, S. K., and Guan, J. L. (2020). FAK signaling in cancerassociated fibroblasts promotes breast cancer cell migration and metastasis by exosomal miRNAs-mediated intercellular communication. Oncogene 39, 2539-2549. doi: 10.1038/s41388-020-1162-2

Wu, Q., Li, B., Li, Z., Li, J., Sun, S., and Sun, S. (2019a). Cancer-associated adipocytes: key players in breast cancer progression. J. Hematol. Oncol. 12:95.

Wu, Q., Li, J., Li, Z., Sun, S., Zhu, S., Wang, L., et al. (2019b). Exosomes from the tumour-adipocyte interplay stimulate beige/brown differentiation and reprogram metabolism in stromal adipocytes to promote tumour progression. J. Exp. Clin. Cancer Res. 38:223.

Wu, Q., Sun, S., Li, Z., Yang, Q., Li, B., Zhu, S., et al. (2018). Tumour-originated exosomal miR-155 triggers cancer-associated cachexia to promote tumour progression. Mol. Cancer 17:155.

Xu, C. G., Yang, M. F., Ren, Y. Q., Wu, C. H., and Wang, L. Q. (2016). Exosomes mediated transfer of IncRNA UCA1 results in increased tamoxifen resistance in breast cancer cells. Eur. Rev. Med. Pharmacol. Sci. 20, 4362-4368.

Yan, W., Wu, X., Zhou, W., Fong, M. Y., Cao, M., Liu, J., et al. (2018). Cancer-cell-secreted exosomal miR-105 promotes tumour growth through the MYC-dependent metabolic reprogramming of stromal cells. Nat. Cell Biol. 20, 597-609. doi: 10.1038/s41556-018-0083-6

Yang, M., Chen, J., Su, F., Yu, B., Su, F., Lin, L., et al. (2011). Microvesicles secreted by macrophages shuttle invasion-potentiating microRNAs into breast cancer cells. Mol. Cancer 10:117.

Yang, S. J., Wang, D. D., Li, J., Xu, H. Z., Shen, H. Y., Chen, X., et al. (2017). Predictive role of GSTP1-containing exosomes in chemotherapy-resistant breast cancer. Gene 623, 5-14. doi: 10.1016/j.gene.2017.04.031

Yang, Y., Li, C. W., Chan, L. C., Wei, Y., Hsu, J. M., Xia, W., et al. (2018). Exosomal PD-L1 harbors active defense function to suppress $\mathrm{T}$ cell killing of breast cancer cells and promote tumor growth. Cell Res. 28, 862-864. doi: 10.1038/s41422018-0060-4

Zhang, P., Wu, X., Gardashova, G., Yang, Y., Zhang, Y., Xu, L., et al. (2020). Molecular and functional extracellular vesicle analysis using nanopatterned 
microchips monitors tumor progression and metastasis. Sci. Transl. Med. 12:eaaz2878. doi: 10.1126/scitranslmed.aaz2878

Zhang, W., Xu, J., Fang, H., Tang, L., Chen, W., Sun, Q., et al. (2018). Endothelial cells promote triple-negative breast cancer cell metastasis via PAI-1 and CCL5 signaling. FASEB J. 32, 276-288. doi: 10.1096/fj.201700237rr

Zhao, L., Gu, C., Gan, Y., Shao, L., Chen, H., and Zhu, H. (2020). Exosomemediated siRNA delivery to suppress postoperative breast cancer metastasis. J. Control. Release 318, 1-15. doi: 10.1016/j.jconrel.2019.12.005

Zheng, Z., Chen, M., Xing, P., Yan, X., and Xie, B. (2019). Increased expression of exosomal AGAP2-AS1 (AGAP2 Antisense RNA 1) in breast cancer cells inhibits trastuzumab-induced cell cytotoxicity. Med. Sci. Monit. 25, 2211-2220. doi: $10.12659 / \mathrm{msm} .915419$

Zhou, W., Fong, M. Y., Min, Y., Somlo, G., Liu, L., Palomares, M. R., et al. (2014). Cancer-secreted miR-105 destroys vascular endothelial barriers to promote metastasis. Cancer Cell 25, 501-515. doi: 10.1016/j.ccr.2014. 03.007
Conflict of Interest: The authors declare that the research was conducted in the absence of any commercial or financial relationships that could be construed as a potential conflict of interest.

Publisher's Note: All claims expressed in this article are solely those of the authors and do not necessarily represent those of their affiliated organizations, or those of the publisher, the editors and the reviewers. Any product that may be evaluated in this article, or claim that may be made by its manufacturer, is not guaranteed or endorsed by the publisher.

Copyright (c) 2021 Wang, Sun, Huang, Li, Fu, Li and Yin. This is an open-access article distributed under the terms of the Creative Commons Attribution License (CC BY). The use, distribution or reproduction in other forums is permitted, provided the original author(s) and the copyright owner(s) are credited and that the original publication in this journal is cited, in accordance with accepted academic practice. No use, distribution or reproduction is permitted which does not comply with these terms. 